\title{
Systematic identification of synergistic combinations of targeted agents and immunotherapies in breast cancer using intratumor multiplex implantable microdevice assay
}

\section{Zuzana Tatarova \\ Harvard Medical School \\ Dylan Blumberg \\ Oregon Health \& Science University \\ James Korkola \\ Oregon Health and Science University \\ Laura Heiser}

Department of Biomedical Engineering, Oregon Health Sciences University https://orcid.org/00000003-3330-0950

\section{John Muschler}

Oregon Health \& Science University

\section{Pepper Schedin}

Oregon Health and Science University https://orcid.org/0000-0003-4244-987X

\section{Sebastian Ahn}

Harvard Medical School

\section{Gordon Mills}

Oregon Health and Science University https://orcid.org/0000-0002-0144-9614

\section{Lisa Coussens}

Oregon Health Sciences University https://orcid.org/0000-0003-2389-1865

\section{Oliver Jonas}

Harvard Medical School

Joe Gray ( $\sim$ grayjo@ohsu.edu )

Oregon Health and Science University https://orcid.org/0000-0001-9225-6756

\section{Article}

Keywords: breast cancer, synergistic combinations, long-term breast cancer control

Posted Date: November 24th, 2021 
DOI: https://doi.org/10.21203/rs.3.rs-1054728/v1

License: (a) (i) This work is licensed under a Creative Commons Attribution 4.0 International License. Read Full License

Version of Record: A version of this preprint was published at Nature Biotechnology on July 4th, 2022. See the published version at https://doi.org/10.1038/s41587-022-01379-y. 


\section{Systematic identification of synergistic combinations of targeted agents and}

2 immunotherapies in breast cancer using intratumor multiplex implantable

3 microdevice assay

5 Zuzana Tatarova, ${ }^{1,2,3}$ Dylan C. Blumberg, ${ }^{1}$ James E. Korkola, ${ }^{1,2}$ Laura M. Heiser, ${ }^{1,2}$ John L.

6 Muschler, ${ }^{1,2}$ Pepper J. Schedin, ${ }^{2,4}$ Sebastian W. Ahn, ${ }^{3}$ Gordon B. Mills,,${ }^{5}$ Lisa M. Coussens, ${ }^{2,4}$

7 Oliver Jonas,,$^{3, *}$ Joe W. Gray ${ }^{1,2, *}$

$8{ }^{1}$ Department of Biomedical Engineering, OHSU Center for Spatial Systems Biomedicine

$9 \quad{ }^{2}$ Knight Cancer Institute

10 Oregon Health \& Science University, Portland, OR 97239, USA

11 '3epartment of Radiology, Brigham \& Women's Hospital

12 Harvard Medical School, Boston, MA 02115, USA

$13{ }^{4}$ Department of Cell and Developmental Biology

$14{ }^{5}$ Division of Oncologic Sciences

15 Oregon Health \& Science University, Portland, OR 97239, USA

$16 *$ Correspondence: ojonas@harvard.bwh.edu (O.J.), grayjo@ohsu.edu (J.W.G.)

\section{Abstract}

19 Systematically identifying synergistic combinations between targeted agents and

20 immunotherapies in cancer based on genomic or other static biomarkers remains elusive. Here

21 we integrate two novel high-content and high-throughput techniques, an implantable

22 microdevice to administer multiple drugs into different sites in tumors at nanodoses; and spatial

23 systems analysis of tumor microenvironmental states to describe tumor cell and immunological

24 response signatures and rapidly, within days, identify effective combinations from among

25 numerous agents. We demonstrate in systemic follow-up studies across three mammary

26 carcinoma models that combinations identified by this approach lead to highly synergistic

27 effects. Biomarkers associated with resistance to each agent allowed us to prioritize at least five

28 novel treatment strategies of which the panobinostat/venetoclax/anti-CD40 was the most

29 effective inducing complete tumor control across models. We show that spatial association of 
cancer stem cells with dendritic cells during immunogenic cell death is a potential mechanism of

31 action underlying long-term breast cancer control.

\section{Introduction}

34 Modern cancer therapies increasingly seek to effect tumor control by simultaneously attacking tumor intrinsic vulnerabilities, enhancing anti-tumor immune activity and/or mitigating stromal mediators of resistance. Targeted drugs typically are designed to attack genetic or transcriptional vulnerabilities on which tumor cells depend for survival but non-malignant cells do not ${ }^{1}$. Genomic screening approaches have supported such tumor-intrinsic aspects of precision medicine, leading to matching of genomic aberrations with specific targeted agents ${ }^{2}$. In breast cancer, treatments targeting tumors that depend on estrogen receptor (ER) signaling, aberrant signaling resulting from human epidermal growth factor receptor 2 (HER2) amplification and/or over expression, CDK4/6 signaling and defects in DNA repair in triple negative breast cancer (TNBC) have been particularly effective ${ }^{3}$. Unfortunately, these treatments are not uniformly effective even in primary tumors carrying the target and are usually only transiently effective in metastatic disease $e^{4,5}$. This may be due in part to drug modulation of aspects of the tumor microenvironment (TME) suggesting that treatment efficacy can be increased by combing these drugs with agents that increase immunogenicity and/or counter microenvironment-mediated resistance, a hypothesis that we address in this paper.

The concept of enhancing cancer treatment efficacy by combining chemotherapies and targeted drugs with agents that enhance immune mediated anti-tumor activity is increasingly well established ${ }^{6}$. The clearest example is the use of immunotherapies, including immune checkpoint blocking (ICB) antibodies as complements to tumor targeted therapies in various liquid and solid malignancies ${ }^{7}$. However, many cancers do not benefit from ICB including in breast cancer where efficacy has been limited to a subset of TNBC patients ${ }^{8,9}$. This lack of efficacy has been attributed, in part, to low tumor mutational burden ${ }^{10}$ and to two mechanisms: i) Low antigenicity

56 through decreased expression of major histocompatibility complex class I (MHC-I) proteins observed mainly in luminal $\mathrm{ER}+\mathrm{BC}^{4,11}$ and $\mathrm{HER} 2+\mathrm{BCs}^{12,13}$; and ii) a naturally immunosuppressive TME associated mainly with TNBC and HER2+ $\mathrm{BC}^{14,15}$. Both of these

59 mechanisms may limit CD8+ $\mathrm{T}$ cell-mediated anti-tumor responses, which then cannot be

60 leveraged to improve efficacy of ICB therapies ${ }^{16}$. Combinations of conventional chemotherapies 
61 and/or targeted anticancer drugs that increase immunogenic cell kill promise significant improvements in overall outcome ${ }^{17,18}$. However, further understanding of drug-immune system interactions is required to design effective and safe immune modulating combinatorial regimens.

64 A variety of experimental approaches have been deployed to elucidate the effects of drug 65 combinations on the tumor and stromal components and to identify biomarkers that inform on 66 the efficacy of treatment combination decisions ${ }^{1}$. Biomarkers typically are identified by

67 establishing associations between tumor features and outcomes in clinical studies ${ }^{10}$ such as those 68 supported by the NCI National Clinical Trials Network ${ }^{19}$, The Cancer Genome Atlas ${ }^{20}$ and

69 Human Tumor Atlas Network ${ }^{21}$ programs. However, these association-based approaches need to

70 be tested for causality in systems that faithfully recreate the interactions of the various

71 components of the TME. Common model systems include tumors that arise in immune competent mice and short- or long-term ex vivo cultures comprised of tumor and stromal

73 components using miniscule scaffolds and active fluidics to closely model specific aspects of the

$74 \mathrm{TME}^{22,23}$. However, the whole animal mouse studies typically are slow, expensive and labor75 intensive, and comprehensive modeling and faithful recapitulation of TME interactions in ex 76 vivo systems remains a major challenge ${ }^{24}$.

77 We report now on an integrated in vivo approach to rapidly, safely and efficiently assess the 78 effects of multi-drug treatments on the TME composition and architecture in living mice. Our 79 study focuses on mouse mammary cancers and our approach is based on the intratumor delivery 80 of nanoliter doses (nanodoses) of multiple drugs or drug combinations into spatially separate 81 regions of a tumor using a minimally invasive, implantable microdevice (IMD) $)^{25-27}$ and 82 multiplexed immunohistochemical (mIHC) assessments ${ }^{28,29}$ of the in-situ responses of the tumor83 microenvironment milieu near each drug delivery site. Computational analyses of serial mIHC 84 staining and imaging of $30+$ proteins allow precise characterization of tumor cell states (e.g. 85 proliferation, stemness, antigenicity, apoptosis) as well as comprehensive classification of cells 86 comprising the TME including immune cells, vasculature and other stroma cells. Assessment of 87 the composition and spatial distribution of the functionally different cell types in each drug 88 delivery area facilitates identification of drug-mediated mechanisms of response and resistance 89 that suggest new therapeutic interventions. We refer to this approach as the Multiplex 90 Implantable Microdevice Assay (MIMA) and we used it to evaluate the effects of five targeted 91 anticancer agents (olaparib, palbociclib, venetoclax, panobinostat, lenvatinib) and two 
92 chemotherapies (doxorubicin, paclitaxel) to predict synergistic anti-tumor effects with different

93 immune-based therapies. The data predicted that palbociclib would synergized with anti-CSF1R,

94 venetoclax with anti-CD40 and panobinostat with anti-PD-1 immunotherapy, respectively, which

95 we validated in traditional systemic dosing studies. We found the triple combination of

96 panobinostat, venetoclax and anti-CD40 as curative and well-tolerated across multiple models of

97 mammary cancer with immunogenic cell death and spatial association of dendritic cells with

98 cancer stem cells representing the likely mechanism underlying cancer stem cell specific anti-

99 tumor immunity in breast cancer.

100

\section{Results}

102 MIMA components and design

103 The IMD used for drug delivery in the MIMA system was a $5 \mathrm{~mm}$ long, $0,75 \mathrm{~mm}$ diameter

104 biocompatible resin cylinder that delivered multiple drugs or drug combinations in up to 18

105 spatially separate regions inside a living tissue (Fig. 1a). IMDs were loaded with drugs

106 formulated with poly-ethylene glycol (PEG) in semi-solid form so that drugs are released with

107 controlled kinetics upon implantation via passive diffusion ${ }^{25}$. Local concentrations of drugs in

108 the IMD were tuned to produce drug levels at each site in the tissue that recapitulate those

109 achieved during systemic treatment (Extended Data Fig. 1a and Supplementary Table 1).

110 Importantly, the nanodoses of drugs do not generate the whole animal toxicities typically

111 associated with systemic treatments ${ }^{25}$.

112 After treatment for 3 days, tumors were harvested with the IMD in place, prepared as a formalin

113 fixed, paraffin embedded (FFPE) samples and serial tissue sections were cut orthogonal to the

114 axis of the IMD (Fig. 1a). Sections through each drug delivery well were stained using $\mathrm{mIHC}-\mathrm{a}$

115 process of serial immunostaining, imaging and stripping (Fig. 1b and Extended Data Fig. 1b,

116 c) ${ }^{28,29}$ - to assess local drug effects using a range of markers with specific staining patterns being

117 cross validated against those generated using cyclic immunofluorescence (cycIF) ${ }^{28}$ (Extended

118 Data Fig. 1c-f). The-mIHC generated multiprotein images were then analyzed by segmenting

119 individual cells and calculating protein expression levels in each segmented cell (Fig. 1c and

120 Extended Data Fig. 2). For our MIMA studies we developed a comprehensive mouse specific

121 readout panel including $30+$ proteins (Fig. 1d and Supplementary Table 2, 3) with the criteria to 
122 (i) interrogate a broad range of tumor and TME states and functions and (ii) to identify

123 actionable phenotypes with preferential detection of early and local responses. We selected 13

124 proteins (Epcam, CD45, CD31, aSMA, CD3, CD4, CD8, CD11b, F4/80, CSF1R, CD11c, Ly6G,

125 MHC-II; Fig. 1a, baseline discovery panel) to classify 17 "standard cell types" which were

126 essential and satisfactory to capture major TME states predicting effective treatment

127 combinations (Fig. 1e-g and Supplementary Table 4). We interrogated additional proteins to

128 refine the 17 standard cell types and/or to report on basic drug sensitivity (proliferation,

129 apoptosis), immunogenic cell death and/or processes typically associated with resistance such as

130 cancer stem cells (Fig. 1d; extended readout).

\section{MIMA identifies drug specific histological signatures of TME response predicting rational}

\section{treatment combinations}

134 We used the MIMA system to perform a small-scale in situ screen and quantitatively assess 135 responses to seven FDA approved drugs with distinct modes of action. The targeted drugs were

136 the poly (adenosine diphosphate [ADP]) ribose polymerase (PARP) inhibitor, olaparib; the

137 multi-kinase vascular endothelial growth factor receptor (VEGFR)-1/2/3 inhibitor, lenvatinib; the

138 cyclin dependent kinase (CDK)-4/6 inhibitor, palbociclib; the B-cell lymphoma (BCL)-2

139 inhibitor, venetoclax; and the pan- histone-deacetylase (HDAC) inhibitor, panobinostat. The

140 chemotherapeutic drugs were the DNA-intercalating agent, doxorubicin and the mitotic inhibitor,

141 paclitaxel that are often used in first line therapy for $\mathrm{BCs}^{30}$. We assessed the responses in tumors

142 arising in immunocompetent MMTV-PyMT (mouse mammary tumor virus-polyoma middle

143 tumor-antigen) mice - a commonly used genetically engineered mouse model for breast cancer

144 that mirrors many aspects of human breast cancer progression and heterogeneity ${ }^{31,32}$. We chose a

145 spontaneous rather than transplanted tumor model to better account for all stages of immune-

146 biology associated with de novo tumor progression ${ }^{33}$, including editing ${ }^{34}$.

147 Our analyses of harvested tumors focused on the cell and molecular compositions and 148 organizations that were significantly enriched in regions close to the drug delivery sites 149 compared to distant intratumoral controls (Fig. 1h). The changes observed for our 17 standard 150 cell types are summarized in Fig. 1g for all seven drugs and Figs. 1i-1 show computed images of 151 selected cell types after treatment. 
152 Lenvatinib and paclitaxel produced no detectable effects and they resembled those produced by

153 PEG negative control (Fig. 1g, i and Extended Data Fig. 3a-c); while olaparib caused only a

154 modest increase in macrophage, neutrophil and fibroblast number (Fig. 1g). Doxorubicin did not

155 mediate immune changes, but did cause a significant enrichment of endothelial cells (Fig. 1g and

156 Extended Data Fig. 3d) suggesting that normalization of vasculature 35,36 could increase efficacy

157 of doxorubicin in breast cancer. Palbociclib, venetoclax, and panobinostat produced the strongest

158 changes in the immune and non-immune stromal states (Fig. 1g, j, k 1). We extended mIHC

159 analytics and performed spatial cell measurements to describe the mechanism of action of these

160 drugs in more detail.

162 Palbociclib induces enrichment of CSF1R+ macrophages associated with pericyte 163 branching and de novo tumor proliferation

164 Intratumoral treatment with palbociclib induced significant accumulation of several stromal cell

165 types into the assay area including CSF1R-positive, MHC-II negative pro-tumorigenic

166 macrophages $^{6}$, endothelial cells, vascular pericytes and mesenchymal cells (Fig. 1g, j; 2a, b and

167 Extended Data Fig. 4a-c). Spatial analyses measuring relative abundance of cells at increasing

168 distances from the drug delivery well showed that while the CD45+ macrophages - as classified

169 by standard cell type - were localized to regions immediately proximal to the drug delivery

170 well; the CD45- less-differentiated macrophages ${ }^{37,38}$ were localized both proximally and more

171 distally (Fig. 2c, d) and in some regions were associated with contractile pericytes ${ }^{36}$ (Fig. 2d).

172 We also assessed the propensity of specific cell types to cluster together by mapping the

173 locations where 10 or more cells of a defined phenotype occurred together in regions 30, 50 or

$17475 \mu \mathrm{m}$ in diameter (Fig. 2e and Extended Data Fig. 4d). These analyses showed that the CSF1R+

175 macrophages and CD31+ endothelial cell/pericyte structures were organized together in response

176 to palbociclib drug stimulus and did not appear in PEG control tissues (Fig. 2e). The patterns for

177 the CD31+ cell aggregates were branch-like with pericytes integrated within endothelial

178 structures suggestive of large vessel formation and enhanced blood flow/pressure control ${ }^{36}$ (Fig.

179 2e and Extended Data Fig. 4d). The profile plot and distance-based cluster analyses also showed

180 clusters of Ki67-positive neoplastic cells distant from the drug delivery site and proximal to the

181 macrophage-pericyte networks (Fig. 2d, e and Extended Data Fig. 4b, d) indicating that the

182 macrophage-pericyte structures likely contribute to an increase in tumor cell proliferation in local 
183 microculture as summarized schematically in Fig. $2 \mathrm{f}$. These results provide direct evidence of

184 how specific changes in tumor microenvironmental states induced by monotherapy may mediate

185 acquired resistance. The high expression of CSF1R on multiple cell types (Fig. 2c) and the

186 associated increase in Ki67+ tumor cells (Fig. 2d-e) suggested to us that targeting the

187 CSF1/CSF1R axis might enhance palbociclib efficacy by countering CSF1R-mediated processes

188 (Supplementary Table 4).

189 We tested this concept in a systemic study of the EMT6 breast cancer model, by treating mice

190 bearing tumors orthotopically implanted into the mammary fat pads of immunocompetent

191 syngeneic mice with intraperitoneal injections of palbociclib, an anti-CSF1R antibody

192 monotherapy, and a combination of the two. The individual drugs did not affect the rate of tumor

193 growth. However, the combination treatment significantly reduced tumor growth (Fig. 2g). Thus,

194 the efficacy of palbociclib/anti-CSF1R suggested by analyses of responses to intratumoral

195 treatments was confirmed in whole animal experiments.

\section{Venetoclax recruits phenotypically distinct clusters of dendritic cells, immature myeloid} cells and endothelial cells

199 Intratumor treatment with venetoclax resulted in significant recruitment of CD11c + dendritic 200 cells (DCs), immature myeloid cells and CD31+ endothelial cells to the drug assay area (Fig.

201 1g,k; Fig. 3a and Extended Data Fig. 4e, f). Unlike in the palbociclib condition, the CD31+ 202 endothelial cells did not express $\alpha$ SMA suggesting they formed small blood vessels that were 203 not supported by pericytes ${ }^{36}$ (Fig. 3b). CD11c+ DCs, which play a critical role in regulating the

204 balance between immune tolerance and activity ${ }^{39}$, aggregated into multiple, spatially separate 205 clusters in regions near venetoclax delivery, but not in random intratumoral regions far from the 206 drug releasing site (Fig. 3c). The clusters were phenotypically distinct as defined by their 207 morphology (Fig. 3d) and expression of Epcam, CD45, MHC-II and CD11b (Fig. 2e). DCs 208 closer to the reservoir exhibited brighter and smaller nuclei (Fig. 3d, 1) and greater than $60 \%$ 209 were Epcam+, CD45- (Fig. 2e) suggesting that they were phagocytic ${ }^{40}$; while others displayed a 210 "Bull's-eye" membrane CD45 staining pattern typical of unstimulated myeloid cells ${ }^{40}$ (Fig. 3d,

211 4). However, only a small fraction of these cells, which were spatially associated with

212 endothelial cells (Fig. 2d, 3), were MHC-II positive (Fig. 3e) and thus were likely limited in their 
213 ability to present available tumor antigens ${ }^{41}$. Agonist monoclonal anti-CD40 antibodies can act

214 on DCs and immature myeloid cells to increase their antigen presenting capacity, maturation and

215 activation potential (called licensing) thereby shifting the balance from tolerance to anti-tumor

216 immunity ${ }^{39,42,43}$. We reasoned that this immunotherapy could be used to enhance anti-tumor

217 capacity of the immune cells recruited by venetoclax which were already primed to have

218 antitumor activity (Fig. 3f).

219 Our test of this hypothesis by systemic treatment of the E0771 orthotopic breast cancer model

220 with a combination of venetoclax and an anti-CD40 agonist showed that this combination

221 reduced tumor growth rate and increased overall survival with $60 \%$ of mice surviving for $>180$

222 days (Fig. 3g). For comparison, the combination of venetoclax with a programmed death ligand-

2231 (PD-1) inhibitory antibody did not significantly affect tumor growth rate or survival (Fig. 3g).

224 Again, a therapeutic strategy suggested by the MIMA proved to be effective in whole animal

225 experiments.

227 Panobinostat induces immunogenic cell death associated with recruitment of antigen 228 presenting neutrophils and macrophages

229 Intratumor delivery of panobinostat led to significant recruitment of several immune cell 230 populations including dendritic cells, antigen presenting macrophages and (antigen presenting)

231 neutrophils with the latter being the most abundant (Fig. 1g, 1; 4a, b and Extended Data Fig. 5a232 c).

233 Neutrophils are considered to be rapid responders against pathogens and classically are not 234 categorized as professional antigen presenting cells as compared to DCs, B-cells, monocytes and 235 macrophages, which have superior ability to prime naïve $\mathrm{T}$ cells ${ }^{41}$. However, $13 \%$ of neutrophils 236 were MHC-II-positive (Fig. 4c, d) suggesting had undergone strong phenotypic maturation ${ }^{44}$.

237 MHC-II + neutrophils have recently been linked to immunogenic cell death (ICD) during which 238 they phagocytose dying tumor cells and mediate respiratory-burst-dependent cytotoxicity against 239 residual cells ${ }^{44}$. Interestingly, panobinostat induced the highest cell kill among the seven drugs 240 tested (Fig. 4e, f). Based on our observation of significant enrichment of MHC-II+ antigen 241 presenting neutrophils associated with cell death, we hypothesized that panobinostat-mediated 
242 cell death would be immunogenic and the efficacy of this targeted therapy would be enhanced by

243 PD-1 blockade.

244 Systemic treatment of EMT6 and E0771 model tumors with panobinostat plus anti-PD-1

245 increased survival duration and reduced tumor growth rate relative to treatment controls or to

246 treatment with panobinostat alone (Fig. $5 \mathrm{~g}$ ) indicating effective induction of antitumor immunity.

247 Consistent with this, systemic treatment with panobinostat significantly increased the proportion

248 of intratumoral CD8+ T cells as compared to stromal parenchyma (Extended Data Fig. 5d).

249 However, the treatments did not achieve long term tumor control (Fig. 5c), and in vaccination

250 studies ${ }^{45}$, only a subset of mice in both EMT6 and E0771 models rejected the tumor post re-

251 challenge (Fig. 4i). These results suggest that resistance mechanisms exist that might counter the

252 full potential of panobinostat-mediated antitumor immunity, and thus we explored this treatment

253 condition in more detail.

Biomarkers of response and mechanisms of resistance associated with early induced antitumor immunity in breast cancer

257 Through literature review, we generated a list of early in situ biomarkers which have been 258 directly or indirectly linked to ICD, increased tumor CD8+ T cell infiltrate and/or ICB efficacy.

259 These include: intercellular adhesion molecule 1 (ICAM1) ${ }^{46,47}$, myeloperoxidase (MPO) ${ }^{47}$, 260 calreticulin $^{17,18,48}$, MHC-I ${ }^{49,50}$, galectin- $3^{46,51}$, neuropilin- ${ }^{52,53}$ and PD-L1 $1^{8,9}$. We validated the 261 presence of these biomarkers at panobinostat reservoirs (Fig. 5a and Extended Data Fig. 6a) and 262 measured their expression and spatial association in relation with the standard stromal cell types 263 in the assay area (Fig. 5b) as well as cancer stem cells (CSCs, Epcam-CD45+PyMT+Ki67-

264 Sox9+) (Fig. 5c-f) - a subset of tumor cells that have self-renewal and tumor initiating capacity 265 which often exhibit resistance to anti-cancer treatments ${ }^{5,54,55}$.

266 ICAM1, MPO and Neuropilin-1 were localized in the proximal cell death and neutrophils rich

267 assay region; while PD-L1, galectin-3, MHC-I and calreticulin were localized mostly on tumor

268 cells distal from the well with the latter two having decreasing gradient profile (Fig. 5a and

269 Extended Data Fig. 6a). A majority (65\%) of Ly6G+ neutrophils were positive for MPO

270 (Extended Data Fig. 6b) consistent with cytotoxic capacity. Positivity for ICAM1 (Fig. 5b) and

271 the mutually exclusive expression of the immune suppressive molecule arginase- 1 on this 
272 population (Extended Data Fig. 5b and 6a) indicate these are anti-tumor (reported also as N1)

273 rather than protumor (N2) neutrophils ${ }^{56}$. Co-treatment with panobinostat and an anti-Ly6G

274 antibody decreased panobinostat mediated cell death implying that these neutrophils may have

275 tumor-killing function as a result of the drug's mechanism of action (Extended Data Fig. 6c).

276 The vast majority (up to $88 \%$ ) of neuropilin-1 positive cells proximal to the panobinostat well

277 were cytotoxic neutrophils (Fig. 5a and Extended Data Fig. 6d) raising the possibility that

278 neuropilin-1 may be a novel biomarker of anti-tumor neutrophils in $\mathrm{BC}-\mathrm{a}$ hypothesis that

279 remains to be functionally tested.

280 Nuclear expression of Sox9 has been associated with stemness in mammary tissue and mammary 281 carcinoma ${ }^{5,54,55}$. We observed CC3 and nuclear Sox9 staining to be mutually exclusive (Fig. 5c

282 and Extended Data Fig. 5b) at the border of cell death/neutrophil rich region providing direct in

283 vivo evidence that the CSCs were resistant to the most potent tumor killing therapy in our screen.

284 In contrast, galectin-3 and Sox 9 were co-expressed in many areas of the border region (Fig. 5c,

285 d) with $22 \%$ of galectin-3+ cells being CSCs (Fig. 5b). This indicates galectin-3 might be

286 classified as a new biomarker enriching CSCs in breast cancer. Expression and spatial

287 association of galectin-3 with both response (MHC-I, calreticulin) and resistance (PD-L1 and

288 CSCs) mechanisms (Fig. 5a-d) suggest pleiotropic involvement of this protein which implies that

289 targeting galectin-3 during immunogenic cell death should be carefully considered (Extended

290 Data Fig. 6c).

291 Finally, we wanted to measure which immune cells are spatially located within the resistant

292 cancer stem cell niche. Three spatial analyses, including macroscopic profile plots of relative cell

293 abundance (Fig. 5a), as well as the distance-based cluster analyses (Fig. 5d), and pairwise

294 proximity measurements in Sox9 microcultures (Fig. 5e, f and Extended Data Fig. 6e, f) showed

295 that CD11c+ dendritic cells were preferentially located in close proximity to CSCs, suggesting

296 functional interactions between the two cell types.

298 Combination of panobinostat, venetoclax and anti-CD40 immunotherapy maximizes tumor 299 killing and anti-tumor immunity in mammary carcinoma

300 The observed spatial association between CSC and DC allowed us to generate a model of drug 301 response maximizing anti-tumor activity through immune-modulation. In this model (Fig. 6a), 
302 panobinostat induces immunogenic cell death of bulk tumor while CSCs remain resistant in the

303 TME. Venetoclax induces recruitment of DCs that we have shown to localize to the - now

304 accessible - CSC niche. We hypothesize that CD40 ligation induced licensing of DCs that had

305 captured and processed antigen from neighboring CSCs, would result in activation of CSC-

306 specific anti-tumor immunity leading to complete tumor clearance. Thus, panobinostat is

307 presumed to induce antitumor immunity on the level of bulk tumor, while venetoclax/anti-CD40

308 may induce anti-tumor immunity on the level of resistant, tumor initiating cancer stem cells.

309 We tested the possibility that combination of panobinostat/venetoclax (PV) with anti-CD40

310 immunotherapy would provide maximal therapeutic efficacy in breast cancer. We tested this by

311 systemically treating mice bearing EMT6 and E0771 tumors and compared the responses to

312 those obtained using a PV/anti-PD-1 combination. Treatment with PV/anti-PD-1 significantly

313 reduced the tumor burden as compared to dual PV and panobinostat/anti-PD-1 (Fig. 6b, c)

314 treatments with survival rates of $40 \%$ in mice bearing EMT6 tumors (Fig. 6c). The triple

315 combination of PV/anti-CD40, however, was superior and eliminated measurable tumors in

$316100 \%$ of EMT6 tumors and 85\% of E0771 tumors, respectively (Fig. 6c, d). We also assessed the

317 efficacy of PV/anti-CD40 against spontaneous tumors arising in the MMTV-PyMT model and

318 found that this combination inhibited tumor progression and doubled the overall survival (Fig.

319 6d). Importantly, none of the combination treatments in whole animal studies were associated

320 with adverse events, likely because we used lower systemic concentrations of drugs than

321 published previously. While antigen specific $\mathrm{T}$ cell responses remain to be critically evaluated,

322 overall, these results suggest the triple combination of panobinostat, venetoclax and anti-CD40

323 as a highly synergistic therapeutic strategy for long term breast cancer control.

\section{Conclusion}

326 The MIMA platform described here provides a strategy to design effective combination regimens

327 based on intratumor nanodose exposure to a range of agents (Supplementary Table 4), coupled

328 with highly multiplexed phenotyping and integrated spatial analysis of tumor response to each

329 therapy. The focal drug delivery begins at the time of implantation and can be treated as a spatial

330 and temporal pharmacological perturbation. Since distances from the drug delivery wells reflect

331 recruitment events, analyses of the responses produced by devices left in place provide data

332 about drug induced changes in cellular densities, molecular phenotypes and possible functional 
333 cell interactions. These MIMA based observations rapidly translate into models of drug response

334 in which we can identify therapeutic vulnerabilities that can be used to predict effective TME-

335 modulating combination treatment strategies (Fig. 2f,g, 3f, g and 6a). Many of these drug effects

336 are difficult or impossible to study in animal models treated systemically, due to heterogeneous

337 and indeterminate drug distribution that can vary greatly over different regions of a tumor and

338 over time. The TME response patterns obtained from MIMA studies may in future be used as

339 early in situ biomarkers of therapeutic response and their further computational processing could

340 provide actionable information to guide the development of effective drug doses and schedules.

341 By testing multiple therapeutic strategies in the same tumor, we can for the first time perform

342 systems level analysis using multiple parallel pharmacological perturbations in the same

343 organism. Furthermore, recent work by Jonas et al has demonstrated that IMD applications are

344 safe and feasible in patients across multiple cancer indications including breast, prostate, $\mathrm{T}$ cell

345 lymphoma and glioblastoma ${ }^{27}$. It may become feasible to use the MIMA approach to measure

346 multiple drug responses in individual patients to guide their combination treatment design.

347 Although intended as proof of concept that analyses of local nanodose drug responses can

348 effectively guide systemic treatment strategies, we have already identified specific therapeutic

349 strategies that warrant clinical consideration. Based on local, significantly enriched

350 histopathological signatures, we predicted synergies of palbociclib with anti-CSF1R, venetoclax

351 with anti-CD40, panobinostat with anti-PD-1 monoclonal antibody and doxorubicin with

352 vasculature-normalizing agents (not validated here). These strategies have been tested in only a

353 few model systems and more extensive testing in subtype specific manner is warranted.

354 However, the combination of lower dose panobinostat/venetoclax/anti-CD40 appears especially

355 effective in three different models and so should be considered for clinical evaluation.

356 All in all, MIMA represents a new approach to identification of effective combination regimens

357 for individual patients on a personalized basis. Extended use of MIMA will also open new

358 opportunities in in silico modeling to model dynamic drug-tumor-stromal interactions.

360 Acknowledgements

361 We thank the OHSU Histopathology Share Resources core (Todd Camp, Joscelyn Zarceno and

362 Cheyenne Martin) for careful FFPE processing of the MIMA tumor samples; Young Hwan 
363 Chang, Sam Sivagnanam, Eugene Manley and AeSoon Bensen for help with image processing

364 and mIHC/cycIF staining; Tiziana Cotechini for sharing the LPA3 mouse model line; and

365 Marilyn McWilliams and Dottie Waddell breast cancer advocates for valuable feedback during

366 project reports. This work was supported by the Susan G. Komen grant SAC190012 (JWG), the

367 NIH/NCI Cancer Systems Biology Consortium Center U54CA209988 (JWG), and the NIH grant

368 R01CA223150 (OJ, LMC).

\section{Author contributions}

371 Conceptualization, ZT, OJ, JWG; Methodology, ZT, OJ, JWG; Software, ZT, DCB;

372 Investigation, ZT, DCB; Data Analysis and Interpretation, ZT, OJ, JWG; Writing - original and 373 final draft, ZT, OJ, JWG; Writing - review \& editing, JEK, LMH, JLM, PJS, SWA, GBM,

374 LMC; Resources, JLM, LMC, OJ, JWG; Funding, OJ, JWG; Supervision, OJ, JWG.

\section{Declaration of interests}

377 J.E.K. is a cofounder and stock holder of Convergent Genomics.

378 GBM has licensed technologies to Myriad Genetics and Nanostring; is on the SAB or is a 379 consultant to Amphista, AstraZeneca, Chrysallis Biotechnology, GSK, ImmunoMET, Ionis, 380 Lilly, PDX Pharmaceuticals, Signalchem Lifesciences, Symphogen, Tarveda, Turbine, and 381 Zentalis Pharmaceuticals; and has stock/options/financial interests in Catena Pharmaceuticals, 382 ImmunoMet, SignalChem, and Tarveda.

383 LMC is a paid consultant for Cell Signaling Technologies, Shasqi Inc., and AbbVie Inc.; 384 received reagent and/or research support from Janssen Research \& Development, LLC, Abbott 385 Labs, Eisai, Inc., ImClone, Aduro Biotech, Inc., Becton Dickinson, Plexxikon Inc., 386 Pharmacyclics, Inc., Acerta Pharma, LLC, Deciphera Pharmaceuticals, LLC, Genentech, Inc., 387 Roche Glycart AG, Syndax Pharmaceuticals Inc., Innate Pharma, and NanoString Technologies, 388 and Cell Signaling Technologies; and is a member of the Scientific Advisory Boards of Syndax 389 Pharmaceuticals, Carisma Therapeutics, Zymeworks, Inc, Verseau Therapeutics, Cytomix 390 Therapeutics, Inc., and Kineta Inc., Hibercell, Inc., Cell Signaling Technologies, Alkermes, Inc. 391 O.J. is a consultant to Kibur Medical. Dr. Jonas's interests were reviewed and are managed by 392 BWH and Mass General Brigham in accordance with their conflict of interest policies. 
393 JWG has licensed technologies to Abbott Diagnostics, PDX Pharmaceuticals and Zorro Bio; has 394 ownership positions in Convergent Genomics, Health Technology Innovations, Zorro Bio and 395 PDX Pharmaceuticals; serves as a paid consultant to New Leaf Ventures; has received research 396 support from Thermo Fisher Scientific (formerly FEI), Zeiss, Miltenyi Biotech, Quantitative 397 Imaging, Health Technology Innovations and Micron Technologies; and owns stock in Abbott 398 Diagnostics, AbbVie, Alphabet, Amazon, AMD, Amgen, Apple, Berkshire, Cisco systems, 399 Clorox, Colgate Palmolive, Crown Castle Int., Humana, Keysight, Linde, Proctor and Gamble, 400 Qualcomm, Unilever, Gilead, Intel, Johnson \& Johnson, Microsoft, Nvidia, Taiwan 401 Semiconductor, and Zimmer Biomet.

402 The other authors declare no competing interests.

404 Methods

405 Murine Models

406 Mice were purchased from The Jackson Laboratory. All animal studies were conducted in 407 accordance with protocols approved by Institutional Animal Care and Use Committee (IACUC) 408 at OHSU (protocol number: IP00000956). All mice were bred and housed under specific 409 pathogen free conditions under a standard $12 \mathrm{~h}$ light / $12 \mathrm{~h}$ dark cycle. C57LB/6, BALB/c, and $410 \mathrm{FVB} / \mathrm{N}$ mice were purchased from the Jackson Laboratory. MMTV-PyMT were from Dr. Lisa 411 Coussens and purchased from the Jackson Laboratory. Virgin female mice of 8-24 weeks of age 412 were used for all experiments.

414 Cell lines

415 EMT6 (mouse breast cancer) cells were purchased from American Type Culture Collection and 416 were maintained in Waymouth's medium with 10\% FBS, and 2mM L-glutamine. E0771 (mouse 417 breast cancer) cells were purchased from $\mathrm{CH} 3$ BioSystems ${ }^{\circledR}$ and were cultured in RPMI-1640 418 with 10\% FBS and 10mM HEPES. Both cell lines were pathogen tested and were grown at 5\% $419 \mathrm{CO}_{2}$ and $37 \mathrm{C}$. 
422 The objective of the studies in figures is to show how intact tumor microenvironment responds to

423 local stimulus of drug release and to test whether this response was significantly different from

424 the baseline tumor microenvironmental state in tumor region distant from the drug site. The

425 number of independent biological replicates of each experiment (n) performed are given in the

426 figure legends. Spatial systems analyses were designed to quantitatively define directional spatial

427 cell dependencies and cause consequence cell association with distance from the reservoir

428 translating to models of drug response. Within these models we aimed to identify therapeutic

429 vulnerabilities to predict rational immune or TME modulating treatment combinations and their

430 optimal schedule/sequencing which we then validated in traditional whole animal studies.

\section{Microdevice implantation studies and sample collection}

433 Nanodose drug delivery devices were manufactured and implanted as described previously in ${ }^{25}$.

434 Briefly, cylindrical microdevices $5.5 \mathrm{~mm}$ in length and $750 \mu \mathrm{m}$ in diameter were manufactured

435 from medical-grade Delrin acetyl resin blocks (DuPont) by micromachining (CNC

436 Micromachining Center) with 18 reservoirs $200 \mu \mathrm{m}$ (diameter) x $250 \mu \mathrm{m}$ (depth) on the outer

437 surface. Reservoirs were packed with drugs mixed with Polyethylene glycol (PEG, MW 1450,

438 Polysciences) polymer at the concentrations indicated in Table S1. Recommended systemic dose

439 in cancer patients was derived from the https://rxlist.com web page to June 2017. Systemic doses

440 ranging between $0-1 \mathrm{mg} / \mathrm{kg}, 1-2 \mathrm{mg} / \mathrm{kg}, 2-4 \mathrm{mg} / \mathrm{kg},>4 \mathrm{mg} / \mathrm{kg}$ translate to $20 \%, 25 \%, 30 \%$ and $40 \%$

441 of drug concentration in PEG, respectively, when released from the nanowell. The calibration

442 was determined previously using mass spectrometry measurements (Jonas et al., 2015). Pure

443 PEG was used in control conditions. Implanting multiple devices per tumor and/or multifocal

444 animal model can increase the throughput up to 50-70 times as compared to conventional

445 systemic treatment studies. When two drugs were loaded into one reservoir, they were at

446 approximately 1:1 ratio. The combination partner was loaded on the bottom of the well;

447 panobinostat was released first. Microdevices were implanted for three days in MMTV-PyMT

448 with late stage spontaneously growing tumors in all experiments. Tumor size was between 1.2 -

$4491.5 \mathrm{~cm}$ in the longest dimension at the time of implant. Tumors were excised at three days after

450 device implantation unless otherwise stated, fixed for $48 \mathrm{~h}$ in $10 \%$ formalin or $4 \%$

451 paraformaldehyde, then perfused with paraffin. Specimen were sectioned using a standard

452 microtome and $5 \mu \mathrm{m}$ tissue sections were collected from each reservoir. Dry FFPE tissues were 
453 baked in a $65^{\circ} \mathrm{C}$ oven for 30mins. Following deparaffinization with xylene and rehydration in

454 serially graded alcohol to distilled water, slides were subjected to endogenous peroxidase

455 blocking in fresh $3 \% \mathrm{H}_{2} \mathrm{O}_{2}$ for 10 minutes at RT. Sections were then stained by multiplex

456 immunohistochemistry and/or cyclic immunofluorescence (see also Extended Data Fig. $2 \mathrm{~b}$ and 457 c).

\section{Cyclic Immunofluorescence}

460 Before iterative cycles of (i) staining, (ii) whole slide scanning and (iii) fluorophore bleaching, 461 the slides were subjected to heat-mediated antigen retrieval immersed in citrate buffer $(\mathrm{pH} 5.5$, 462 HK0809K, BioGenex Laboratories Citra Plus Antigen Retrieval), then in Tris/EDTA buffer (pH 463 9.0, S2368, Dako Target Retrieval Solution) using Cuisinart Electric Pressure Cooker (CPC464 600N1) for total of 35 to 40 minutes. Protein blocking was performed for 30 minutes RT with $46510 \%$ normal goat serum (S-1000, Vector Lab) and 1\% bovine serum albumin (BP1600-100) in 466 1xPBS. (i) Slides were incubated with primary antibody (concentrations defined in Table S2) for 4672 hours at RT while being protected from light in a dark humid chamber. All washing steps were 468 performed for $3 \times 2-5$ minutes in 1xPBS while agitating. Slides were mounted with SlowFade 469 Gold antifade mountant with DAPI (S36938) using a Corning Cover Glass (2980-245). (ii) 470 Images were acquired using Zeiss Axio Scan.Z1 Digital Slide Scanner (Carl Zeiss Microscopy) 471 at 20x magnification after which the coverslips were gently removed in 1xPBS while agitating.

472 (iii) Fluorophores were chemically inactivated using a $3 \% \mathrm{H}_{2} \mathrm{O}_{2}$ and $20 \mathrm{mM} \mathrm{NaOH}$ in $1 \times \mathrm{PBS}$ for 47330 minutes at RT while being continuously illuminated. The fluorophore inactivation was 474 repeated twice with a short, 10-minute, 1xPBS wash in between. Efficacy of bleaching was 475 imaged before antibody incubation (baseline autofluorescence) and every third to fourth cycle in 476 average. After protein blocking, samples were subjected to the next round of staining. Single cell 477 feature extraction was not applied to evaluate sections stained by cyclic immunofluorescence.

\section{Multiplex Immunohistochemistry}

480 Before iterative cycles of (i) staining, (ii) whole slide scanning and (iii) and heat and chemical 481 stripping of antibodies and chromogen, the slides were subjected to staining with F4/80 and 482 CSF1R antibodies (cycle zero, no antigen retrieval, Supplementary Table 2) and hematoxylin 483 staining (S3301, Dako) for 1-5mins followed by whole slide scanning. Slides were then 
484 subjected to the first heat-mediated antigen retrieval in 1x pH 5.5-6 citrate buffer (Biogenex

485 Laboratories, HK0809K) for 90 seconds in a low power microwave and 16 minutes in a steamer

486 followed by protein blocking with 10\% normal goat serum (S-1000, Vector Lab) and 1\% bovine

487 serum albumin (BP1600-100) in 1xPBS for 30 minutes RT. (i) Slides were incubated with

488 primary antibodies (concentrations defined in Table S2) for 1 hour at RT or 16-17 hours at 4

489 degrees Celsius while being protected from light in a dark humid chamber. Signal was visualized

490 with either anti-rabbit or anti-rat Histofine Simple Stain MAX PO horseradish peroxidase (HRP)

491 conjugated polymer (Nichirei Biosciences) followed by peroxidase detection with 3-amino-9-

492 ethylcarbazole (AEC). Two or three drops of HRP polymer were used for up to nickel-size or

493 whole slide tissue sample, respectively. Timing of AEC development was determined by visual

494 inspection of positive control tissue (Extended Data Fig. 1d-f) for each antibody. All washing

495 steps were performed for 3 x 5-10 minutes in 1xPBS while agitating. Slides were mounted with a

496 filtered 1xPBS with 0.075\% Tween20 (BP337100) using a Signature Series Cover Glass cover

497 glass (Thermo Scientific, 12460S). (ii) Images were acquired using the Aperio ImageScope AT

498 (Leica Biosystems) at 20x magnification after which the coverslips were gently removed in

$4991 \times$ XPB while agitating. (iii) Within one cycle, removal of AEC and HRP inactivation was

500 accomplished by incubating the slides in $0.6 \%$ fresh $\mathrm{H}_{2} \mathrm{O}_{2}$ in methanol for 15 minutes; AEC

501 removal and stripping of antibodies was accomplished by Ethanol gradient incubation and heat-

502 mediated antigen retrieval such as described above between cycles. After washing and protein

503 blocking, samples were subjected to the next round of staining.

Image processing and feature extraction of mIHC images

506 The iteratively digitized images were co-registered using Matlab (The MathWorks, Inc., Natic,

507 MA, version 2019b) utilizing the detectSURFFeatures algorithm from the Computer Vision

508 Toolbox. The imperfectly registered images were additionally processed using the Linear Stack

509 Alignment with SIFT plugin (Fiji) so that cell features overlap down to a single pixel level.

510 Hematoxylin-stained images were color deconvoluted for single cell nuclear segmentation to

511 generate a binary mask using watershed function and standard image processing steps (noise

512 removal, erosion, dilation; Fiji) ${ }^{57}$. AEC chromogenic signal was extracted using the NIH plugin

513 RGB_to_CMYK to separate AEC signal into the yellow channel for improved sensitivity of IHC

514 evaluation ${ }^{58,59}$. Gray scale images of all proteins and the binary mask were imported to 
515 CellProfiler (version 3.1.8, Broad Institute) ${ }^{60}$ to quantify single cell signal mean intensity as

516 defined by mask which was scaled to a range 0-1. IdentifyPrimaryObjects module was used to

517 identify nuclei from mask; MeasureObjectIntensity module measured mean intensity for each

518 object for each protein. The mean signal intensity per cell output was imported to FCS Express 6

519 and 7 Image Cytometry Software (DeNovo Software) to perform multidimensionality reduction

520 to classify "cell standards". Gating strategies and hierarchical cell classification is presented in

521 Fig. 1e and Extended Data Fig. 2e. Polygonal gates moving around central vertex without

522 changing the polygon shapes was used to obtain quantitatively reproducible multiplex data, batch

523 to batch, independent of the condition measured. Positive control tissues were used to help to

524 define single parameter threshold for positivity by manual gating. Total of 3000-5000 cells were

525 analyzed for feature extraction in the assay area located above the drug releasing site with \pm 300

526 total cells for paired, experimental vs control, region. Minimum population proportion within 5\%

527 margin of error and 95\% confidence level was set to $0.75 \%$ (represents 12 cells) to discriminate

528 noise from specific cell enrichment induced by e.g. increased protein expression or cell

529 recruitment into the assay region. Experimental condition of the assay area was compared to

530 random control intratumoral region located perpendicular and/or far from the drug-releasing

531 reservoir. To obtain greater control over cofounding variables, paired sample one tailed t-tests

532 were used to determine enrichment of induced TME states. Percentage of positivity and

533 significance was presented in form of a heatmap or bar graphs. Quality of the single cell data was

534 ensured by excluding deformed (folded), lost or unevenly stained tissue (border effects). The

535 assay area was determined by the first 3000-5000 cells above the well excluding these deformed

536 regions. Single cell data from FCS Express was extracted in data grid to Matlab for downstream

537 spatial systems analyses. In computed images, neutrophils are presented independent of the

538 Epcam \pm status.

540 Spatial Systems Analyses

541 Distance based cluster function finds clusters in a set of spatial points expressed in XY space

542 (adapted and modified from Yann Marcon; Matlab October 2019). The clustering is based on

543 Euclidean distance between the points (cells). The function does not require the number of

544 clusters to be known beforehand. Each cell clusters with the closest neighboring cell if distance

545 between the two cells is shorter than the defined threshold. Minimal number of cells per cluster 
are defined by user. The function outputs non-clustering cells in gray color while each cluster meeting the defined parameters (minimal number of cells within maximum distance range) are presented in randomized colors. Clusters within the maximum defined distance merge and share one color. Number of clusters and total coverage in the assay area was calculated using distinct cluster sizes (defined by minimal number of cells within maximum distance range) for control PEG and palbociclib which identified that cells cluster in response to treatment if minimum 10 cells are present within maximum distance rage 30-75 $\mu \mathrm{m}$ (systematic comparison not shown in this study). Cluster parametrization using as few as 5 cells and distances as large as $100 \mu \mathrm{m}$ resulted in treatment non-specific cluster formation in PEG negative control. Treatment specific cluster formation with cluster definition of minimum 10 cells within $50 \mu \mathrm{m}$ distance was generalizable to all marker and standard cell types which was confirmed in panobinostat condition by comparing assay area and distal region side by side in one field of view (Extended Data Fig. 6e). This treatment specific cluster parametrization was applied in downstream analytics to identify hotspots/zones of interest (e.g. proximal, border, distal, network adjacent, CD11c+ DC clusters) in an objective, biology driven, manner.

561 For the relative abundance profile plot, marker positive cells and the standard cell types were 562 extracted to XY coordinate space, signal was blurred using Gaussian Blur filter and relative 563 abundance of positive cells was displayed with distance from the well in a profile plot as outlines

564 in corresponding Extended Data Figures. A moving average filter with $50 \mu \mathrm{m}$; and $100 \mu \mathrm{m}$

565 window size (movmean function; Matlab) was additionally applied to smoothen the feature 566 signal for palbociclib and panobinostat condition, respectively. Signal in the profile plots was not 567 scaled.

568 Inside the hotspot, spatial (geographical) interactions between marker positive cells were 569 determined by proximity measurements in local microculture by using the pdist 2 function in 570 Matlab (MathWorks, Inc., Natic, MA, version 2019b) which returns the distance of each pair of 571 observations (positive cells) in $\mathrm{X}$ and $\mathrm{Y}$ using metric specified by Euclidean distance. Random 572 circular regions of $175 \mu \mathrm{m}$ diameter (defined by Extended Data Fig. 6f) were selected in the 573 border, cancer stem cell, zone of the panobinostat assay area and Euclidean distance was 574 measured between Sox9+ and other marker positive cells. The number of distances was 575 presented in form of a histogram. To quantify spatially interrelated phenomenon, proportions of 
576 distances lower than $50 \mu \mathrm{m}$ (as defined by distance-based cluster analyses) was compared

577 between different cell pairs (e.g. Sox9+/Ly6G+ vs Sox9+/CD11c+).

578 Extended hierarchical cell classification was applied to characterize the significantly enriched

579 cell phenotypes forming zones of interest which were outside the standard cell type classification

580 (e.g less differentiated macrophages or phagocytic DCs). Probe combination, number of cells

581 analyzed within number of clusters are defined in the figures and figure legends.

582 2D composite and 3D composite images were presented by using Fiji ${ }^{57}$ and QiTissue

583 Quantitative Imaging System (http://www.qi-tissue.com).

584 The spatial systems analyses were used to identify drug models of response (presented as line

585 diagrams) and the identified therapeutic vulnerabilities were tested in whole animal studies.

587 Whole animal treatment studies

588 While the high-throughput IMD experiments were perfomed in the MMTV-PyMT model $31,32,61,62$ 589 with spontanously growing tumors; the whole animal validation studies of predicted immune590 modulating combinations were perfomed using transplantable breast cancer cell lines in 591 syngeneic mice to avoid extensive breeding and colony maintenance necessary to test synergy of 592 multiple predicted combinations. E0771 and EMT6 models, which are typically used in breast 593 cancer research involving immunotherapy testing ${ }^{63-65}$, were selected randomly for validation of 594 different combinatios. The combination of panobinostat and anti-PD-1 was tested in both 595 transplantable models. The most potent triple combination of panobinostat, venetoclax and anti596 CD40 was additionally tested in the MMTV-PyMT model with spontanously growing tumors.

597 MMTV-PyMT transgenic mice that were 80 days old were randomized and included in the study 598 when their total tumor burden was between $150-550 \mathrm{~mm}^{3}$ (treatment initiation). For the 599 orthotopically induced tumor models of mammary carcinoma, EMT6 $\left(0.5 \times 10^{6}\right.$ in $1 \times$ PBS per 600 site), E0771 (0.5 x 10 $10^{6}$ in Corning matrigel per site) and primary tumor derived LPA3 $\left(0.8 \times 10^{5}\right.$ 601 in Corning matrigel per site) cells were injected into the \#4 mammary fat pad of female virgin $602 \mathrm{C} 57 \mathrm{LB} / 6, \mathrm{BALB} / \mathrm{c}$, and FVB/N mice, respectively. One tumor was induced in the E0771, LPA3 603 models and two tumors were induced in the EMT6 model. Caliper measurements were used to 604 calculate the tumor volumes using formula length $\mathrm{x}$ width ${ }^{2} / 2$. Treatments were initiated when 605 total tumor burden was between $60-150 \mathrm{~mm}^{3}$. For all models, the endpoint was determined by 
606 tumor volume above $2000 \mathrm{~mm}^{3}$ in two consecutive measurements or one measurement above

$6072200 \mathrm{~mm}^{3}$. Treatments were administered by intraperitoneal injection. Dose, schedule and

608 duration are indicated in the respective figures and figure legends. Treatment schedule was

609 estimated depending on the location of the targetable cell phenotype in proximity to the well or

610 more distal from the drug source. E.g. cells in the immediate proximity to the drug well at 3 days

611 of exposure were likely recruited first to the drug assay area thus early targeting (pre-treatment)

612 of these cells is preferred. Inversely, cells located in distal regions should be targeted by

613 posttreatment approach. Diluent and IgG2a isotype control (BioXCell) concentrations were

614 equivalent to the highest dose of the respective drug used in each experiment.

615 The mice were monitored daily to determine any possible effects on the general condition of the

616 animals using parameters as established by (Morton and Griffiths, 1985). The guidelines for

617 pain, discomfort and distress recognition were used to evaluate weight loss, appearance,

618 spontaneous behavior, behavior in response to manipulation and vital signs. Specifically, general

619 appearance (dehydration, missing anatomy, abnormal posture, swelling, tissue masses, prolapse)

620 skin and fur appearance (discoloration, urine stain, pallor, redness, cyanosis, icterus, wound,

621 sore, abscess, ulcer, alopecia, ruffled fur), eyes (exophthalmos, microphthalmia, ptosis, reddened

622 eye, lacrimation, discharge, opacity), feces (discoloration, blood in the feces, softness/diarrhea),

623 locomotor (hyperactivity, coma, ataxia, circling) were monitored to determine loss of body

624 condition (BC) score, namely: BC 1 (emaciated) score applied when skeletal structure was

625 extremely prominent with little or no flesh/muscle mass and vertebrae was distinctly segmented;

626 BC 2 (under-conditioned) score applied when segmentation of vertebrate column was evident,

627 dorsal pelvic bones were readily palpable and muscle mass was reduced; BC 3 (well-

628 conditioned) applies when vertebrae and dorsal pelvis were not prominent/visible, and were

629 palpable with slight pressure. Loss of BC was also considered when anorexia (lack or loss of

630 appetite) or failure to drink; debilitating diarrhea, dehydration/reduced skin turgor; edema,

631 sizable abdominal enlargement or ascites, progressive dermatitis, rough hair coat/unkempt

632 appearance, hunched posture, lethargy, loss of righting reflex, neurological signs or bleeding

633 from any orifice appeared in treated mice. Majority of treated groups were well-conditioned (BC

634 score 3); less than $20 \%$ of mice in each group experienced mild diarrhea for up to 2 days once

635 during the course of treatment (typically post first or second therapy administration). Mice

636 receiving palbociclib monotherapy were under-conditioned (BC score 2) starting from day 3 till 
637 the end of the treatment. Two out of eight mice in the MMTV-PyMT model died within 1-3 days 638 after first injection of $\alpha \mathrm{CD} 40$ immunotherapy when administered as single agent. Lethal toxicity 639 of anti-CD40 used as a single agent was previously reported due to a shock-like syndrome ${ }^{66}$ and 640 our data also strongly suggest this immunotherapy is tolerable only with prior administration of 641 anti-cancer agent(s). Surviving mice receiving venetoclax/anti-CD40 combination experienced 642 fur graying to different degree starting approximately four weeks post treatment. No signs of 643 pain, discomfort or distress were observed in the surviving mice. Emaciated (BC score 1), over644 conditioned (BC score 4) nor obese (BC score 5) were observed in our studies.

645 To show CD8+ T cell infiltration inside the tumor bed, ErbB2 $\Delta$ Ex 16 mice ${ }^{67}$ with spontaneously 646 growing late stage tumors were intraperitoneally injected with panobinostat $(15 \mathrm{mg} / \mathrm{mg})$ on day 0 ,

6472 and 4. Tumors were extracted at day 7, were FFPE processed and were stained for CD8 to 648 compare the rate of intratumoral CD8+ T cells in panobinostat treated vs control (diluent) treated 649 mice.

\section{$650 \quad$ Vaccination study}

651 EMT6 and E0771 cells in tissue culture were treated with a soluble drug panobinostat at $5 \mu \mathrm{M}$ 652 concentration when they would reach 60-70\% confluency. After two days the cells were 653 harvested and were injected subcutaneously (total 2-3 x 106 cells) into lower left flank of 654 BALB/c and C57B16 mice, respectively. Cells freeze-thawed three times served as negative 655 control for non-immunogenic form of cell death. After 7-8 days, the mice were re-inoculated by 656 injecting living cells orthotopically into one \#4 mammary fat pad (total $0.5 \times 10^{6}$ cells) and tumor 657 appearance was monitored by minimal tumor size approximately $5 \mathrm{~mm}$ and $3.5 \mathrm{~mm}$ in the longest 658 dimension for E0771 and EMT6 model, respectively (palpable tumors). We note the E0771 659 tumors after re-challenge appeared at the primary subcutaneous site and no tumors were 660 developed in the orthotopic site.

\section{Statistical analysis}

663 All data are combined from two to three independent experiments, unless specifically noted. To 664 accomplish randomization for systemic mouse experiments, animals were sorted by a blinded 665 investigator and then groups were assigned. Each group was checked post-hoc to verify no 666 statistical significance in average starting tumor size. There was no sample-size estimation of in 
667 standard drug treatment experiments. Data are shown as mean $\pm \mathrm{SEM}$, unless otherwise noted.

668 For tumor growth rate, significance was calculated by unpaired two-tailed t-test with equal

669 variance. For survival and tumor free analyses, Kaplan-Meier curves were generated to

670 demonstrate time to event and log-rank (Mantel-Cox) test was used to evaluate statistical

671 significance.

672

673 References

674 1. Letai, A. Functional precision cancer medicine-moving beyond pure genomics. Nat. Med. $675 \quad 23,1028-1035$ (2017).

676 2. Li, A. et al. Characterizing advanced breast cancer heterogeneity and treatment resistance 677 through serial biopsies and comprehensive analytics. npj Precis. Oncol. 5, (2021).

678 3. Hanker, A. B., Sudhan, D. R. \& Arteaga, C. L. Overcoming Endocrine Resistance in $679 \quad$ Breast Cancer. Cancer Cell 37, 496-513 (2020).

680 4. Brady, S. W. et al. Combating subclonal evolution of resistant cancer phenotypes. Nat. $681 \quad$ Commun. (2017) doi:10.1038/s41467-017-01174-3.

682 5. Jeselsohn, R. et al. Embryonic transcription factor SOX9 drives breast cancer endocrine 683 resistance. Proc. Natl. Acad. Sci. U. S. A. 114, E4482-E4491 (2017).

684 6. Kowal, J., Kornete, M. \& Joyce, J. A. Re-education of macrophages as a therapeutic 685 strategy in cancer. Immunotherapy 11, 677-689 (2019).

686 7. Robert, C. A decade of immune-checkpoint inhibitors in cancer therapy. Nat. Commun. $687 \quad 11,10-12(2020)$.

688 8. Adams, S. et al. Current Landscape of Immunotherapy in Breast Cancer: A Review. 689 JAMA Oncol. 5, 1205-1214 (2019).

690 9. Force, J., Leal, J. H. S. \& McArthur, H. L. Checkpoint Blockade Strategies in the 691 Treatment of Breast Cancer: Where We Are and Where We Are Heading. Curr. Treat. 692 Options Oncol. 20, (2019).

693 10. Hugo, W. et al. Genomic and Transcriptomic Features of Response to Anti-PD-1 Therapy 694 in Metastatic Melanoma. Cell 165, 35-44 (2016). 
695 11. Lee, H. J. et al. Differential expression of major histocompatibility complex class I in 696 subtypes of breast cancer is associated with estrogen receptor and interferon signaling. $697 \quad$ Oncotarget 7, 30119-30132 (2016).

698 12. Inoue, M. et al. Expression of mhe class i on breast cancer cells correlates inversely with 699 her2 expression. Oncoimmunology 1, 1104-1110 (2012).

700 13. Janiszewska, M. et al. The impact of tumor epithelial and microenvironmental 701 heterogeneity on treatment responses in HER2+ breast cancer. JCI Insight 6, (2021).

702 14. Denardo, D. G. et al. Functionally Regulates Response to Chemotherapy. Cancer Discov. 703 54-67 (2011) doi:10.1158/2159-8274.CD-10-0028.Leukocyte.

704 15. Gil Del Alcazar, C. R. et al. Immune escape in breast cancer during in situ to invasive $705 \quad$ carcinoma transition. Cancer Discov. 7, 1098-1115 (2017).

706 16. Palucka, A. K. \& Coussens, L. M. The Basis of Oncoimmunology. Cell 164, 1233-1247 $707 \quad$ (2016).

708 17. Galluzzi, L. et al. Molecular mechanisms of cell death: Recommendations of the 709

710 18. Yatim, N., Cullen, S. \& Albert, M. L. Dying cells actively regulate adaptive immune 711 responses. Nat. Rev. Immunol. 17, 262-275 (2017).

712 19. Abrams, J. et al. National Cancer Institute's Precision Medicine Initiatives for the New 713 National Clinical Trials Network. Am. Soc. Clin. Oncol. Educ. B. 71-76 (2014) 714 doi:10.14694/edbook_am.2014.34.71.

715 20. Hutter, C. \& Zenklusen, J. C. The Cancer Genome Atlas: Creating Lasting Value beyond $716 \quad$ Its Data. Cell 173, 283-285 (2018).

717 21. Rozenblatt-Rosen, O. et al. The Human Tumor Atlas Network: Charting Tumor 718 Transitions across Space and Time at Single-Cell Resolution. Cell 181, 236-249 (2020).

719 22. Jenkins, R. W. et al. Ex Vivo Profi ling of PD-1 Blockade Using Organotypic Tumor $720 \quad$ Spheroids. (2017) doi:10.1158/2159-8290.CD-17-0833.

721 23. Tatárová, Z., Abbuehl, J. P., Maerkl, S. \& Huelsken, J. Microfluidic co-culture platform to 722 quantify chemotaxis of primary stem cells. Lab Chip 1-8 (2016) 
doi:10.1039/C6LC00236F.

724 24. Yuki, K., Cheng, N., Nakano, M. \& Kuo, C. J. Organoid Models of Tumor Immunology. Trends Immunol. 41, 652-664 (2020).

726 25. Jonas, O. et al. An implantable microdevice to perform high-throughput in vivo drug sensitivity testing in tumors. Sci. Transl. Med. 7, 284ra57 (2015).

728

729

730

731

732

733

26. Watson, S. S. et al. Microenvironment-Mediated Mechanisms of Resistance to HER2 Inhibitors Differ between HER2 + Breast Cancer Subtypes Article MicroenvironmentMediated Mechanisms of Resistance to HER2 Inhibitors Differ between HER2 + Breast Cancer Subtypes. Cell Syst. 1-14 (2018) doi:10.1016/j.cels.2018.02.001.

27. Dominas, C. et al. The translational and regulatory development of an implantable microdevice for multiple drug sensitivity measurements in cancer patients. 1-10 (2021) doi:10.1109/TBME.2021.3096126.

28. Lin, J.-R., Fallahi-Sichani, M. \& Sorger, P. K. Highly multiplexed imaging of single cells using a high-throughput cyclic immunofluorescence method. Nat. Commun. 6, 8390 (2015).

29. Tsujikawa, T. et al. Quantitative Multiplex Immunohistochemistry Reveals MyeloidInflamed Tumor-Immune Complexity Associated with Poor Prognosis. Cell Rep. 19, $203-$ 217 (2017).

30. Kumar, N., Mb, T. \& Dhesy-Thind Md Msc, S. CLINICAL PRACTICE GUIDELINES IN BREAST CANCER, Kumar Tyagi and Dhesy-Thind Clinical practice guidelines in breast cancer ABSTRACT Background A number of clinical practice guidelines (cpgs) concerning breast cancer (bca) screening and. Curr. Oncol. 25, 151-160 (2018).

31. Guy, C. T., Cardiff, R. D. \& Muller, W. J. Induction of mammary tumors by expression of polyomavirus middle T oncogene: a transgenic mouse model for metastatic disease. Mol. Cell. Biol. 12, 954-961 (1992).

32. Lin, E. Y. et al. Progression to malignancy in the polyoma middle T oncoprotein mouse breast cancer model provides a reliable model for human diseases.Lin, E. Y., Jones, J. G., Li, P., Zhu, L., Whitney, K. D., Muller, W. J., \& Pollard, J. W. (2003). Progression to malignan. Am. J. Pathol. 163, 2113-26 (2003). 
752 33. Hanahan, D. \& Coussens, L. M. Accessories to the Crime: Functions of Cells Recruited to 753 the Tumor Microenvironment. Cancer Cell 21, 309-322 (2012).

754 34. Dunn, G. P., Old, L. J. \& Schreiber, R. D. The Three Es of Cancer Immunoediting. Annu. 755 Rev. Immunol. 22, 329-360 (2004).

756 35. Mpekris, F. et al. Combining microenvironment normalization strategies to improve cancer immunotherapy. Proc. Natl. Acad. Sci. U. S. A. 117, 3728-3737 (2020).

36. Bergers, G. \& Song, S. The role of pericytes in blood-vessel formation and maintenance. Neuro. Oncol. 7, 452-464 (2005).

760 37. Deszo, E. L., Brake, D. K., Cengel, K. A., Kelley, K. W. \& Freund, G. G. CD45 Negatively Regulates Monocytic Cell Differentiation by Inhibiting Phorbol 12-Myristate 13-Acetate-dependent Activation and Tyrosine Phosphorylation of Protein Kinase C $\delta . J$.

38. Norazmi, M. N., Hohmann, A. W., Skinner, J. M. \& Bradley, J. Expression of MHC class II, interleukin 2 receptor and CD45 antigens on tumour-associated tymphocytes in colonic carcinoma. Br. J. Cancer 60, 685-687 (1989).

767 39. Domogalla, M. P., Rostan, P. V., Raker, V. K. \& Steinbrink, K. Tolerance through education: How tolerogenic dendritic cells shape immunity. Front. Immunol. 8, 1-14 (2017).

40. Goodridge, H. S. et al. Activation of the innate immune receptor Dectin-1 upon formation of a "phagocytic synapse". Nature 472, 471-475 (2011).

772 41. Reis E Sousa, C. Dendritic cells in a mature age. Nat. Rev. Immunol. 6, 476-483 (2006).

773 42. Griffiths, K. L. et al. Targeting dendritic cells to accelerate T-cell activation overcomes a bottleneck in tuberculosis vaccine efficacy. Nat. Commun. 7, 1-13 (2016).

775 43. Sotomayor, E. M. et al. Conversion of tumor-specific CD4+ T-cell tolerance to T-cell priming through in vivo ligation of cd40. Nat. Med. 5, 780-787 (1999). sterile immunogenic dying cells drives neutrophil-mediated residual cell killing. Cell Death Differ. 24, 832-843 (2017). 
45. Md Sakib Hossain, D. et al. Dinaciclib induces immunogenic cell death and enhances anti- PD1-mediated tumor suppression. J. Clin. Invest. 128, 644-654 (2018).

782 46. Gittens, B. R., Bodkin, J. V, Nourshargh, S., Perretti, M. \& Cooper, D. Galectin-3: A 783 Positive Regulator of Leukocyte Recruitment in the Inflamed Microcirculation. (2018) doi:10.4049/jimmunol.1600709.

785

786

787 788

789

790

791

792

793

794

795

796

797

798

799

800

801

802

803

804

805

806

807

47. Patnaik, A. et al. Cabozantinib eradicates advanced murine prostate cancer by activating antitumor innate immunity. Cancer Discov. 7, 750-765 (2017).

48. Obeid, M. et al. Calreticulin exposure dictates the immunogenicity of cancer cell death. Nat. Med. 13, 54-61 (2007).

49. Aguilera, T. A. et al. Reprogramming the immunological microenvironment through radiation and targeting Axl. Nat. Commun. Publ. online 23 December 2016; | doil0.1038/ncomms13898 7, 1974-1982 (2016).

50. Luo, N. et al. DNA methyltransferase inhibition upregulates responses in breast cancer. Nat. Commun. 1-11 (2018) doi:10.1038/s41467-017-02630-w.

51. Guerriero, J. L. et al. Class IIa HDAC inhibition reduces breast tumours and metastases through anti-tumour macrophages. Nature (2017) doi:10.1038/nature21409.

52. Chawla, A. et al. Neutrophil elastase enhances antigen presentation by upregulating human leukocyte antigen class I expression on tumor cells. Cancer Immunol. Immunother. 65, 741-751 (2016).

53. Kerros, C. et al. Neuropilin-1 mediates neutrophil elastase uptake and cross-presentation in breast cancer cells. J. Biol. Chem. 292, 10295-10305 (2017).

54. Guo, W. et al. Slug and Sox9 cooperatively determine the mammary stem cell state. Cell 148, 1015-1028 (2012).

55. Xue, Y. et al. SOX9/FXYD3/Src Axis Is Critical for ER + Breast Cancer Stem Cell Function . Mol. Cancer Res. 17, 238-249 (2019).

56. Fridlender, Z. G. et al. Polarization of Tumor-Associated Neutrophil Phenotype by TGF$\beta$ : 'N1' versus 'N2' TAN. Cancer Cell 16, 183-194 (2009).

57. Schneider, C. a, Rasband, W. S. \& Eliceiri, K. W. NIH Image to ImageJ: 25 years of 
image analysis. Nat. Methods 9, 671-675 (2012).

809 58. Pham, N. A. et al. Quantitative image analysis of immunohistochemical stains using a

810 CMYK color model. Diagn. Pathol. 2, 1-10 (2007).

811 59. Banik, G. et al. HHS Public Access. 1-20 (2020) doi:10.1016/bs.mie.2019.05.039.High812 dimensional.

813 60. Carpenter, A. E. et al. CellProfiler: Image analysis software for identifying and

814 quantifying cell phenotypes. Genome Biol. 7, (2006).

815 61. Attalla, S., Taifour, T., Bui, T. \& Muller, W. Insights from transgenic mouse models of PyMT-induced breast cancer: recapitulating human breast cancer progression in vivo. Oncogene 40, 475-491 (2021).

818 62. Varticovski, L. et al. Accelerated preclinical testing using transplanted tumors from genetically engineered mouse breast cancer models. Clin. Cancer Res. 13, 2168-2177 (2007).

821 63. Ewens, A., Mihich, E. \& Ehrke, M. J. Distant metastasis from subcutaneously grown E0771 medullary breast adenocarcinoma. Anticancer Res. 25, 3905-3915 (2005).

64. Rockwell, S. In vivo-in vitro tumour cell lines: characteristics and limitations as models 824 for human cancer. Br. J. Cancer. Suppl. 4, 118-122 (1980).

65. Herschkowitz, J. I. et al. Identification of conserved gene expression features between murine mammary carcinoma models and human breast tumors. Genome Biol. 8, 1-17

66. Van Mierlo, G. J. D. et al. CD40 stimulation leads to effective therapy of CD40- tumors through induction of strong systemic cytotoxic T lymphocyte immunity. Proc. Natl. Acad.

831 67. Turpin, J. et al. The ErbB2 $\Delta \mathrm{Ex} 16$ splice variant is a major oncogenic driver in breast cancer that promotes a pro-metastatic tumor microenvironment. Oncogene 1-12 (2016) doi:10.1038/onc.2016.129. 
Fig. 1

a i

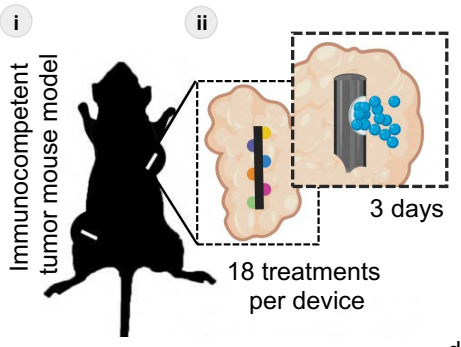

iii

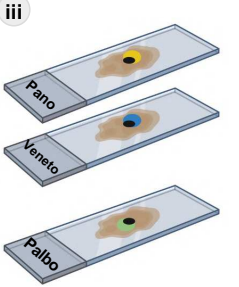

FFPE tissue slice at drug-TME cross-section b

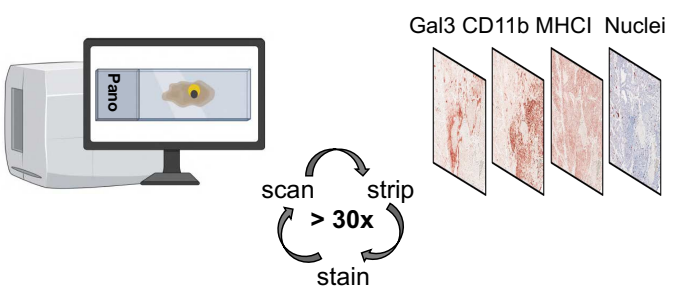

c

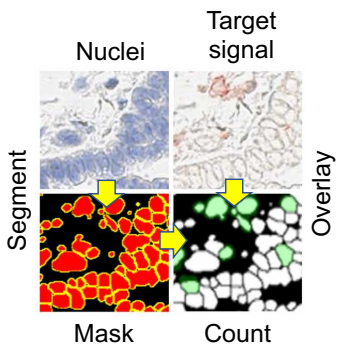

d

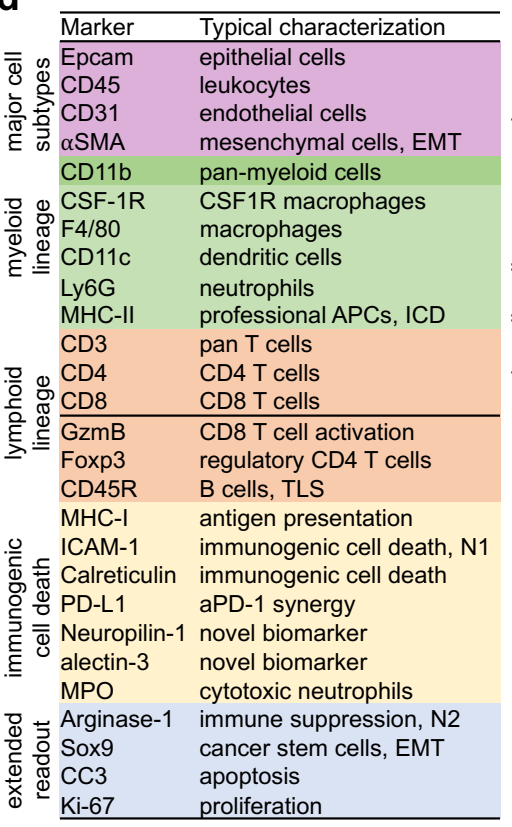

e

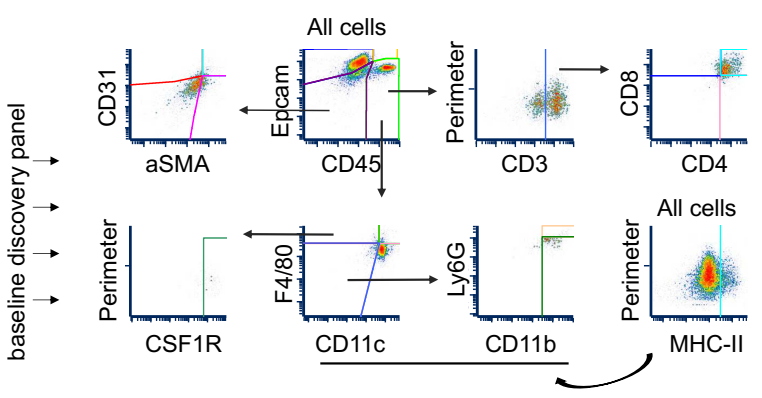

f

\begin{tabular}{ll}
\hline Standard cell type & Identifying probe combination \\
\hline epithelial cells & Epcam+CD45- \\
leukocytes & Epcam-CD45+ \\
T cells & Epcam-CD45+CD3+ \\
CD4 T cells & Epcam-CD45+CD3+CD4+CD8- \\
CD8 T cells & Epcam-CD45+CD3+CD4-CD8+ \\
macrophages (M) & Epcam-CD45+F4/80+CD11c- \\
protumorigenic M & Epcam-CD45+F4/80+CD11c-CSF1R+ \\
antigen presenting M & Epcam-CD45+F4/80+CD11c-CSF1R-MHCII+ \\
dendritic cells (DC) & Epcam-CD45+F4/80-CD11C+ \\
antigen presenting DC & Epcam-CD45+F4/80-CD11c+MHC-II+ \\
neutroprohils (N) & Epcam-CD45+F4/80-CD11C-Ly6G+CD11b+ \\
antigen presenting N & Epcam-CD45+F4/80-CD11c-Ly6G+CD11b+MHCII+ \\
immature myeloid cells & Epcam-CD45+F4/80-CD11c-Ly6G-CD11b+MHCII- \\
endothelial cells & Epcam-CD45-CD31+aSMA- \\
mesenchymal cells & Epcam-CD45-CD31-aSMA+ \\
pericytes & Epcam-CD45-CD31+aSMA+ \\
epcam leukocytes & Epcam+CD45+ \\
\hline
\end{tabular}

g
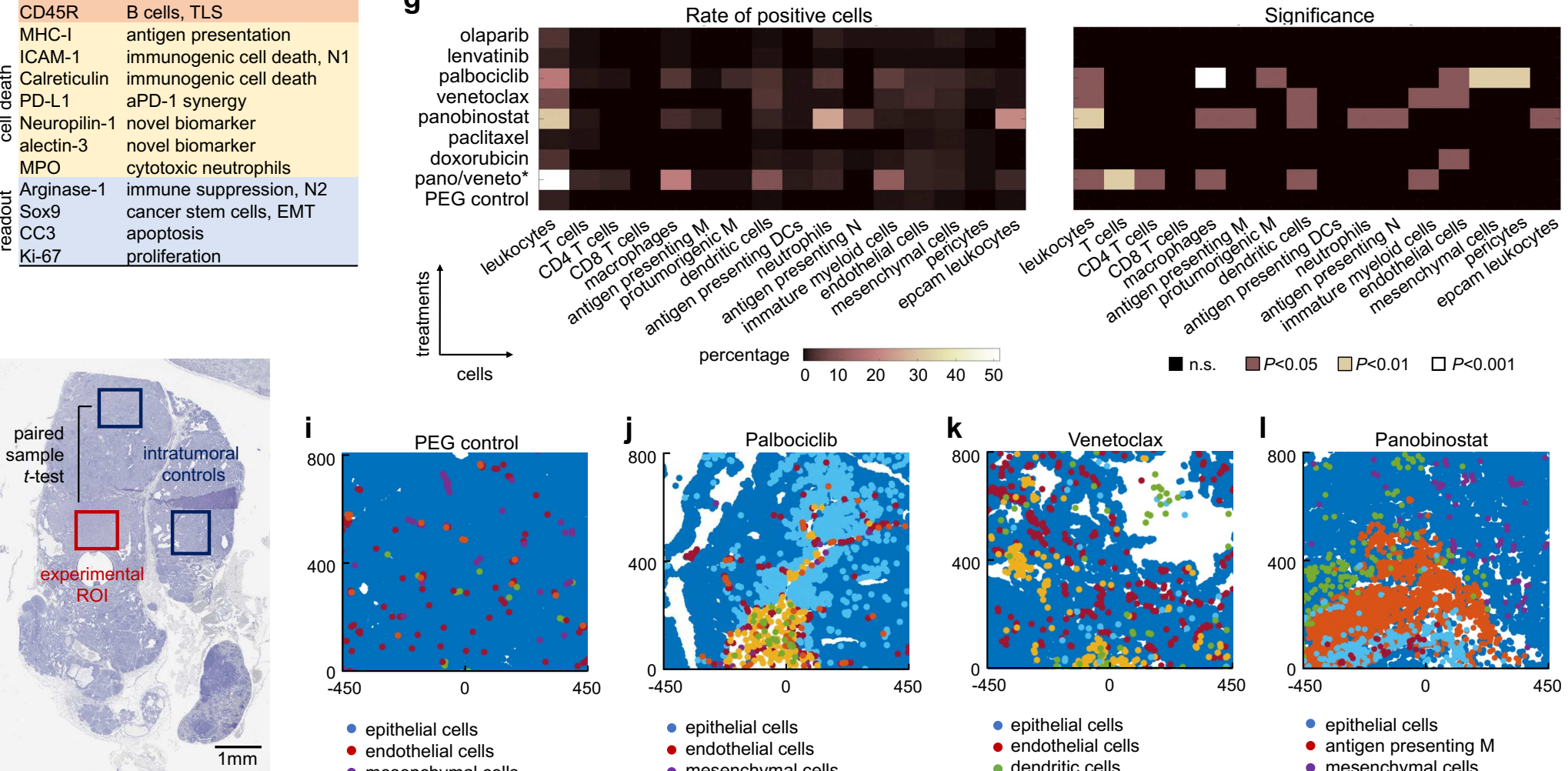

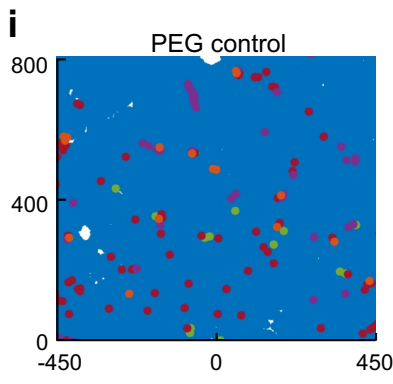

- epithelial cells

- endothelial cells

- mesenchymal cells

- pericytes

- leukocytes

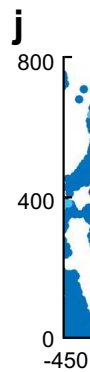

Palbociclib
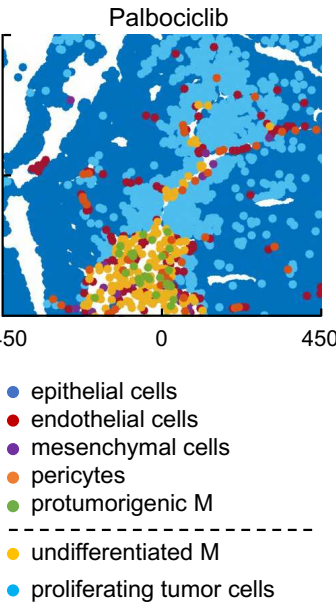

k

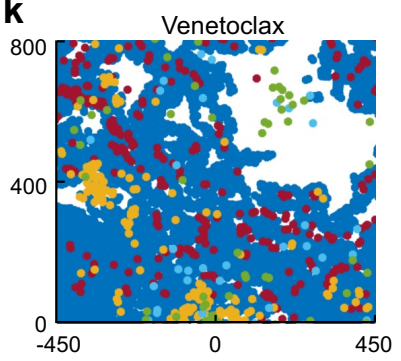

epithelial cells

- endothelial cells

dendritic cells

- immature myeloid cells

phagocytic DCs

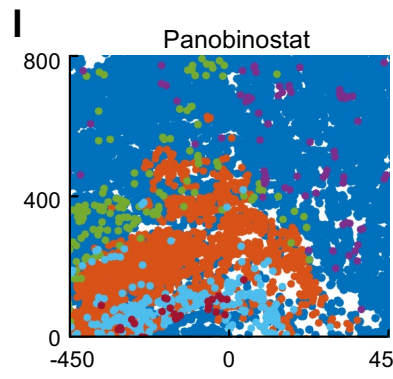

- epithelial cells

- antigen presenting M

- mesenchymal cells

- neutrophils

- antigen presenting $\mathrm{N}$

cancer stem cells

Fig. 1| MIMA components and testing of locally induced drug effects on TME. a, Schematic of IMDs implanted into a multifocal mouse model of mammary carcinoma (i) showing treatments being released into spatially separated regions of tumors through passive diffusion (ii) and each condition being assayed individually (iii). b, Schematic of the $\mathrm{mIHC}$ technique composed of iterative histological stripping, staining and scanning using digital scanning microscopy to detect the target set of markers. c, Acquired images are co-registered with nuclear staining and the mean intensity of antibody staining within a mask is calculated for each cell to count marker positive cells in a spatially intact tissue. $\mathbf{d}$, Antibody list primary probe classification used to interrogate a broad range of tumor intrinsic and tumor-microenvironmental states. e, $\mathbf{f}$, Multidimensionality reduction in hierarchical gating (e) and list of probe combinations identifying standard cell types (f). $\mathbf{g}, \mathbf{h}$, Heatmap of mean percentage of positive cells (left) and level of significance (right) at depicted targeted agents and chemotherapies (y-axis) with PEG being the negative control $(\mathrm{g})$. Total cell counts were between 3000 to 5000 cells per assay area and were matched \pm 300 total cells for paired samples: experimental vs control region as shown in the macroscopic view of the hematoxylinstained tumor tissue implanted with IMD (h). Minimum population proportion within $5 \%$ margin of error and $95 \%$ confidence level was set to $0.75 \%$ (represents 12 cells) to discriminate noise from specific signal. $n=3$ wells from 3 tumors from 2-3 mice per treatment. MMTV-PyMT mice with late stage spontaneously growing tumors were implanted for three days. i-I, Presentation of selected standard cell types in XY space. [0,0] coordinate is the drug releasing site; direction of release is upward. 
a

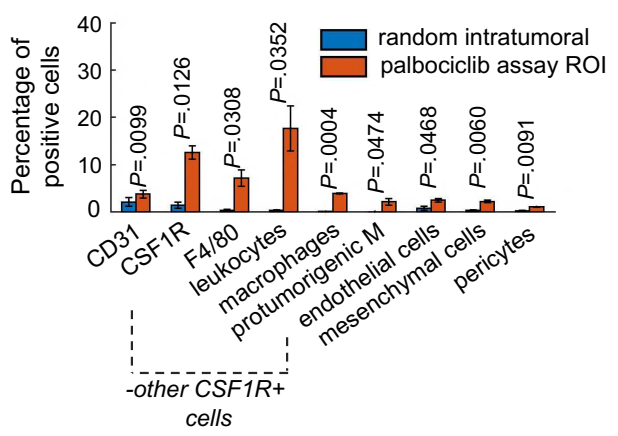

d

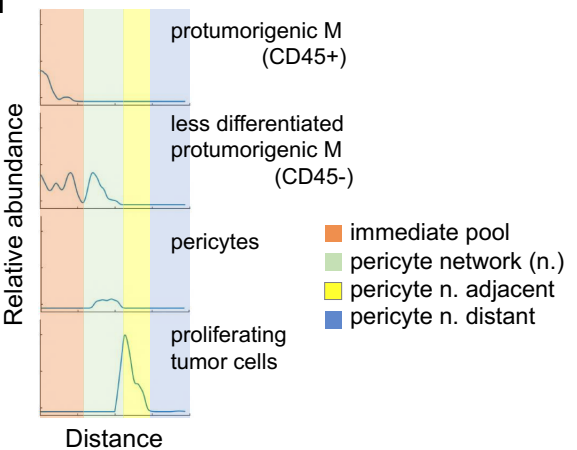

b
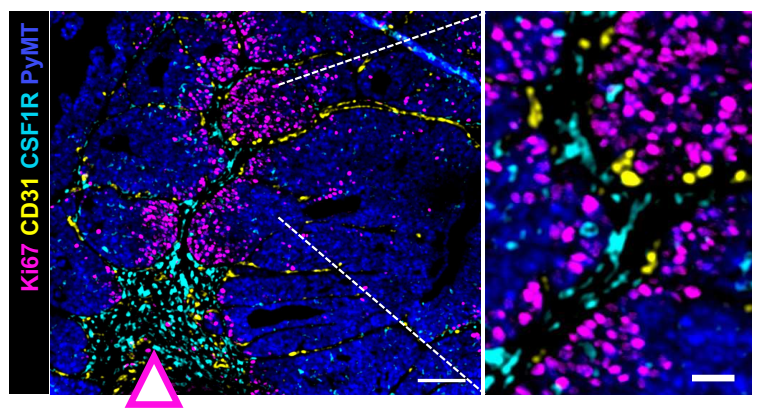

release site
C

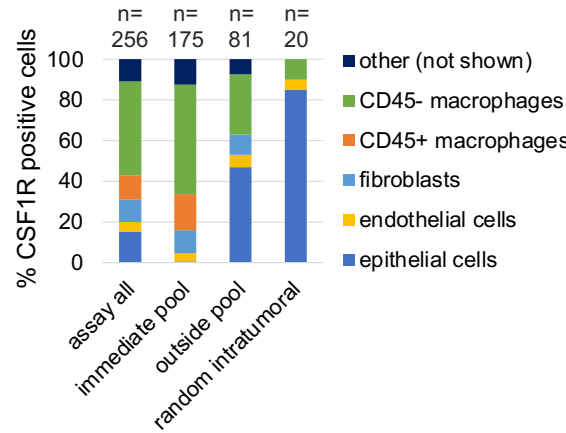

e
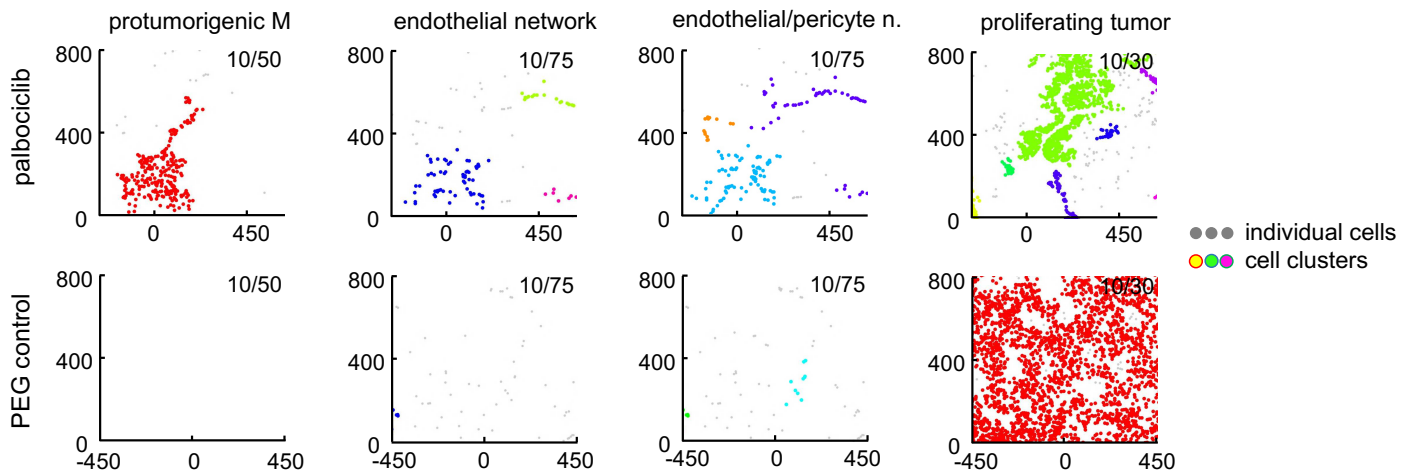

g

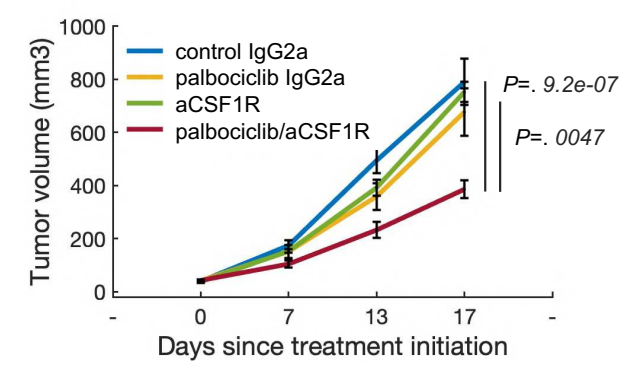

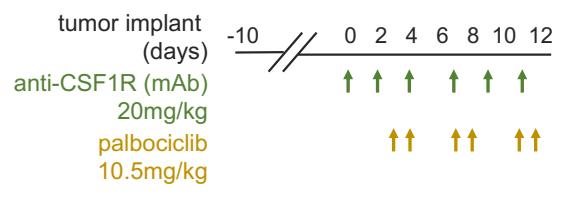

Fig. 2| Local TME changes induced by palbociclib and whole animal studies testing the combination efficacy with predicted anti-CSF1R immunotherapy. a, Quantification of single cell events using individual markers and standard cell type classification. Bars are mean \pm s.e.; $n=3$ reservoirs. Significance was calculated by paired sample one tailed t-test. For quantification of all TME lineages, see Extended Data Fig. 4a.. b, Sample composite image of the key response markers at the palbociclib well. Scale bar is $100 \mu \mathrm{m}$ (left); and $25 \mu \mathrm{m}$ (right). c, Percentage of top five cell types expressing CSF1R stratified by zones in the palbociclib assay area. "Immediate pool" zone is visualized by the dashed line in Extended Data Fig. 4c. The number of cells analyzed $(\mathrm{n})$ is shown. $\mathbf{d}$, Line profile of relative cell abundance as a function of distance from well (left to right). Assay zones are color-coded in the legend; profile line is shown in Extended Data Fig. 4c.. e, Distance-based clustering of depicted cell types as a set of XY coordinates. Coordinate $[0,0]$ identifies the drug source. The direction of the drug release is upward. Clusters were identified by a minimum 10 cells within maximum distances of $50 \mu \mathrm{m}, 75 \mu \mathrm{m}$ and $30 \mu \mathrm{m}$ for CSF1R+ protumorigenic macrophages, endothelial/pericyte network and proliferating tumor cells, respectively. Each cluster is depicted with a randomized color; individual (non-clustering) cells are shown as light gray points. f, Palbociclib model of response presented as line diagram and site of intervention using immunotherapy depicted in red. $\mathbf{g}$, Tumor burden measurement of mice bearing EMT6 tumors after systemic treatment using drugs as color-coded in the graph. Shown is mean \pm s.e.; $n=8$ to 10 tumors per group. Significance was calculated using an independent two-sample two-tailed t-test with equal variance. 
Fig. 3

a

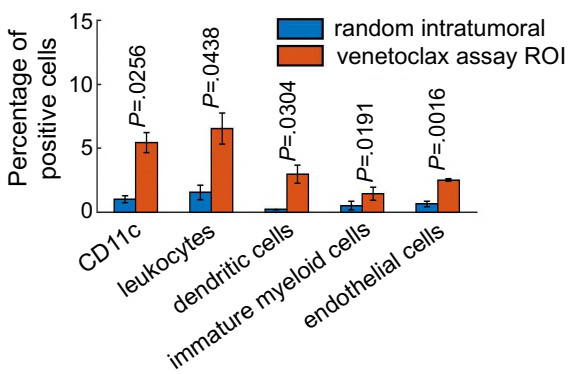

b

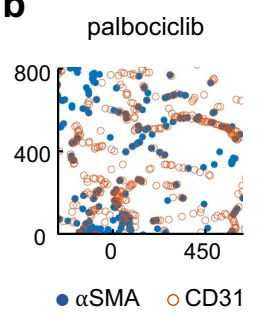

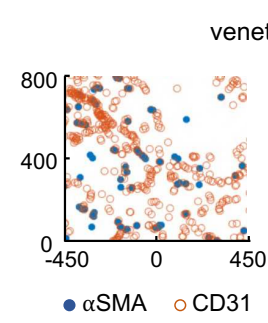

venetoclax

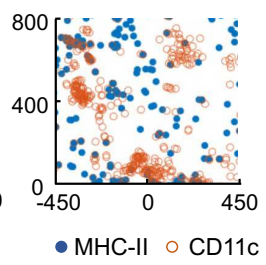

C

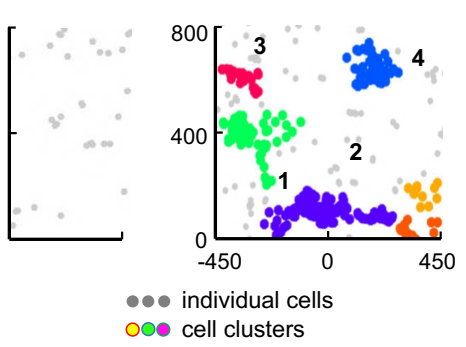

d

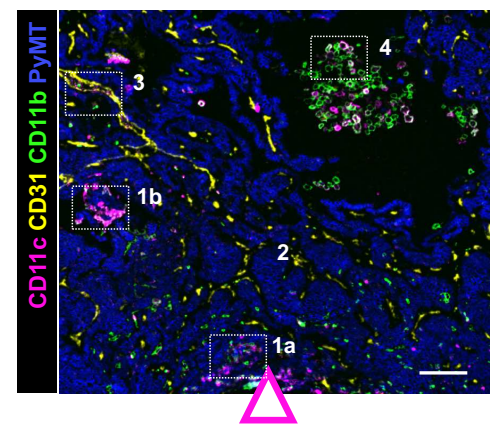

1a, 1b: phagocytic

2: individual

3: migratory (MHC-II+)

4: unstimulated ("bull-eye")
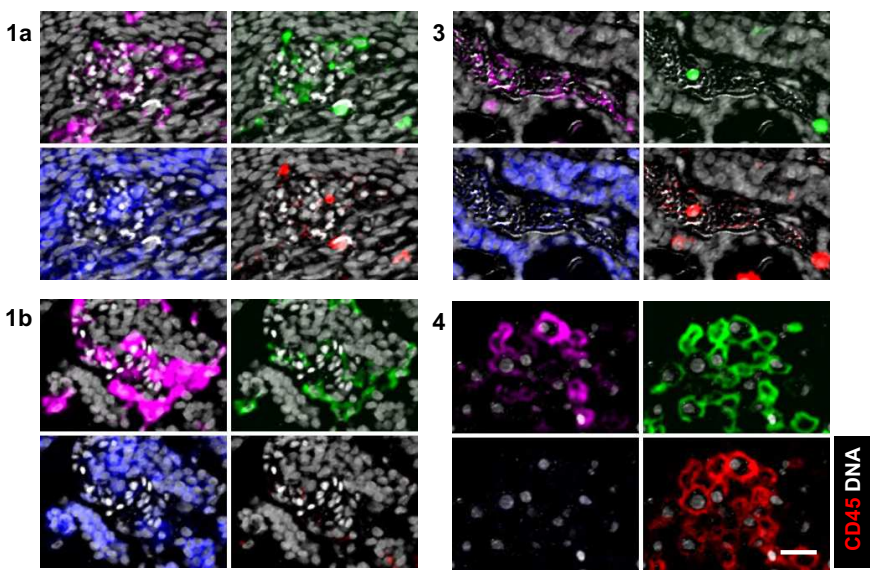

e
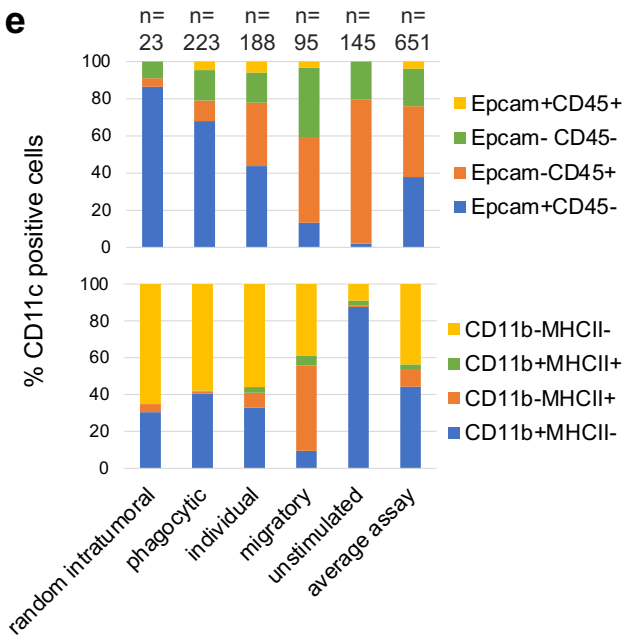

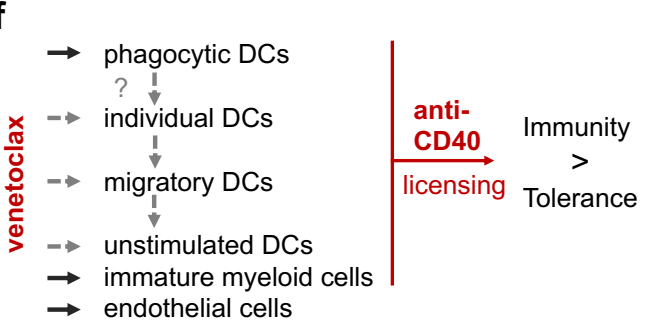

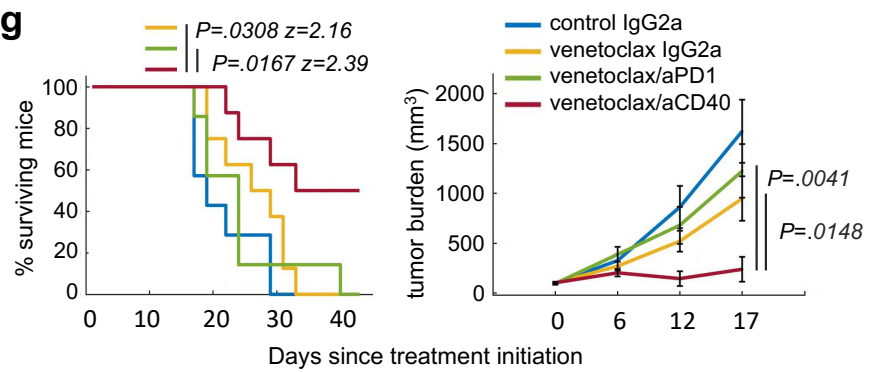

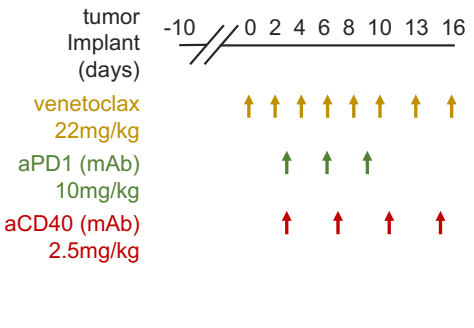

Fig. 3| Local TME changes induced by Venetoclax and whole animal studies testing the combination treatment efficacy with the predicted anti-CD40 immunotherapy. a, Quantification of single cell events using individual markers and standard cell types. Bars are mean $\pm \mathrm{s}$.e.; $\mathrm{n}=3$ reservoirs. Significance was calculated by paired sample one tailed t-test. For quantification of all cells, see Extended Data Fig. 4e. b, Marker coexpression in XY coordinates in the palbociclib (left) and venetoclax (mid, right) assay area. Each color-coded dot represents a marker positive cell. Coordinate $[0,0]$ identifies the drug source. The direction of the drug release is upward. c, Distance-based cluster analysis of CD11c positive cells as a set of XY coordinates in random intratumoral (left) and venetoclax assay (right) regions. Clusters are displayed in randomized colors if at least 10 cells are present within maximum distance range $50 \mu \mathrm{m}$; individual cells not meeting this criterium are shown as light gray points. d, Sample composite image of the key response markers at the venetoclax well. Arrow indicates the source and direction of the drug release. Numbered hashed boxes define the magnified area on the right where individual markers are overlayed on the DNA signal (in white). Scale bar 100 $\mu \mathrm{m}$ (left); and 30 $\mu \mathrm{m}$ (right). e, Percentages of Epcam and CD45 (top) and CD11b and MHC-II (bottom) positive cells within morphologically different CD11c + DCs presented as a stack bar graph. The number of cells analyzed $(n)$ is shown. Two to three ROls from two venetoclax samples were summed per each zone. $f$, Venetoclax model of response presented as an influence diagram with sites of intervention using immunotherapy depicted in red. The relation of morphologically distinct and spatially separate CD11c DC clusters remains unclear (gray dashed arrows). g, Survival rates (left) and tumor burden measurements (right) of mice bearing E0771 tumors after systemic treatment using drugs as color-coded in the line graphs. Shown is mean \pm s.e.; $n=7-8$ mice per group. Significance was calculated by log-rank (Mantel-Cox) and by an unpaired two-tailed t-test with equal variance for survival and tumor burden rate, respectively. For results using anti-PD-1 and anti-CD40 monotherapy see Fig. 6c. 
a

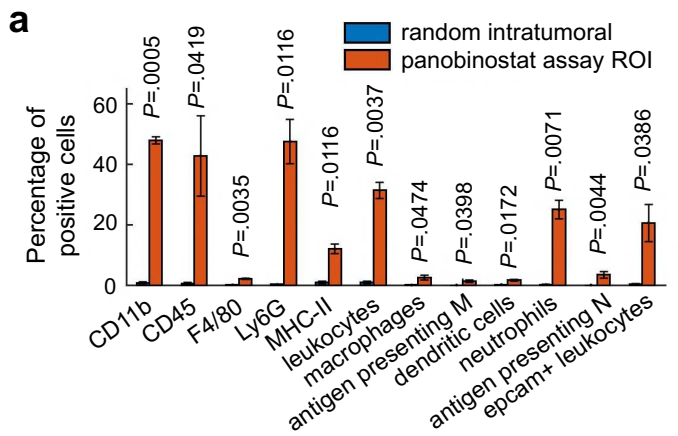

C

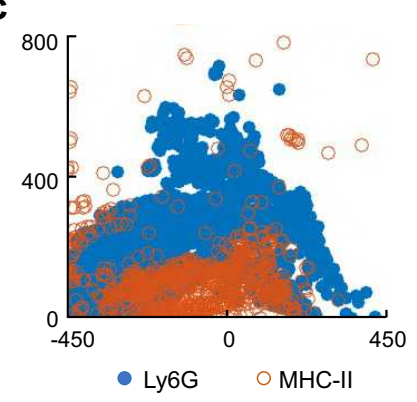

d b

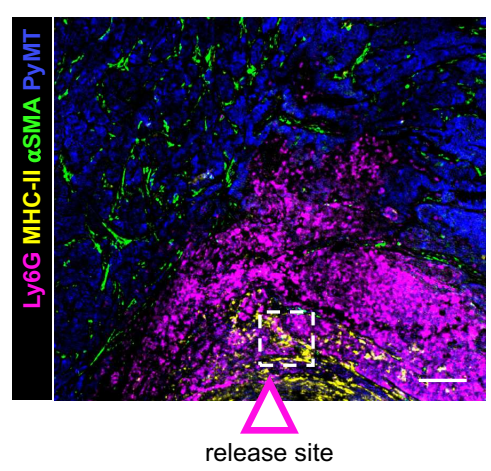

e

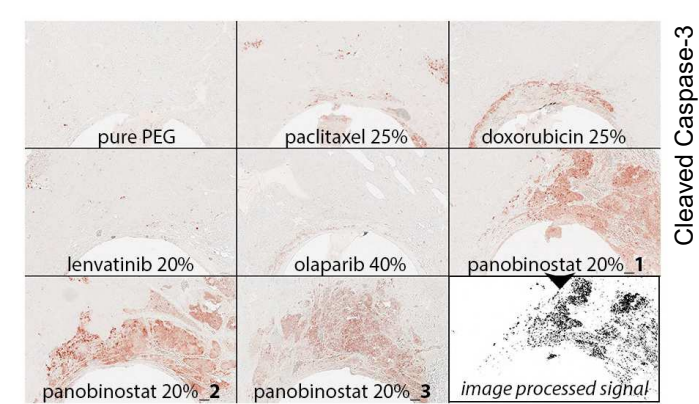

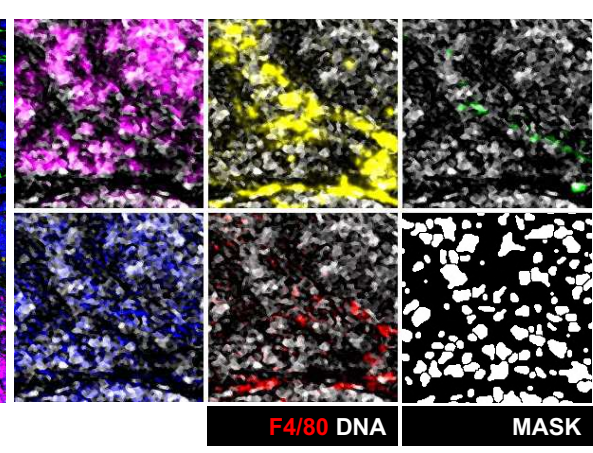
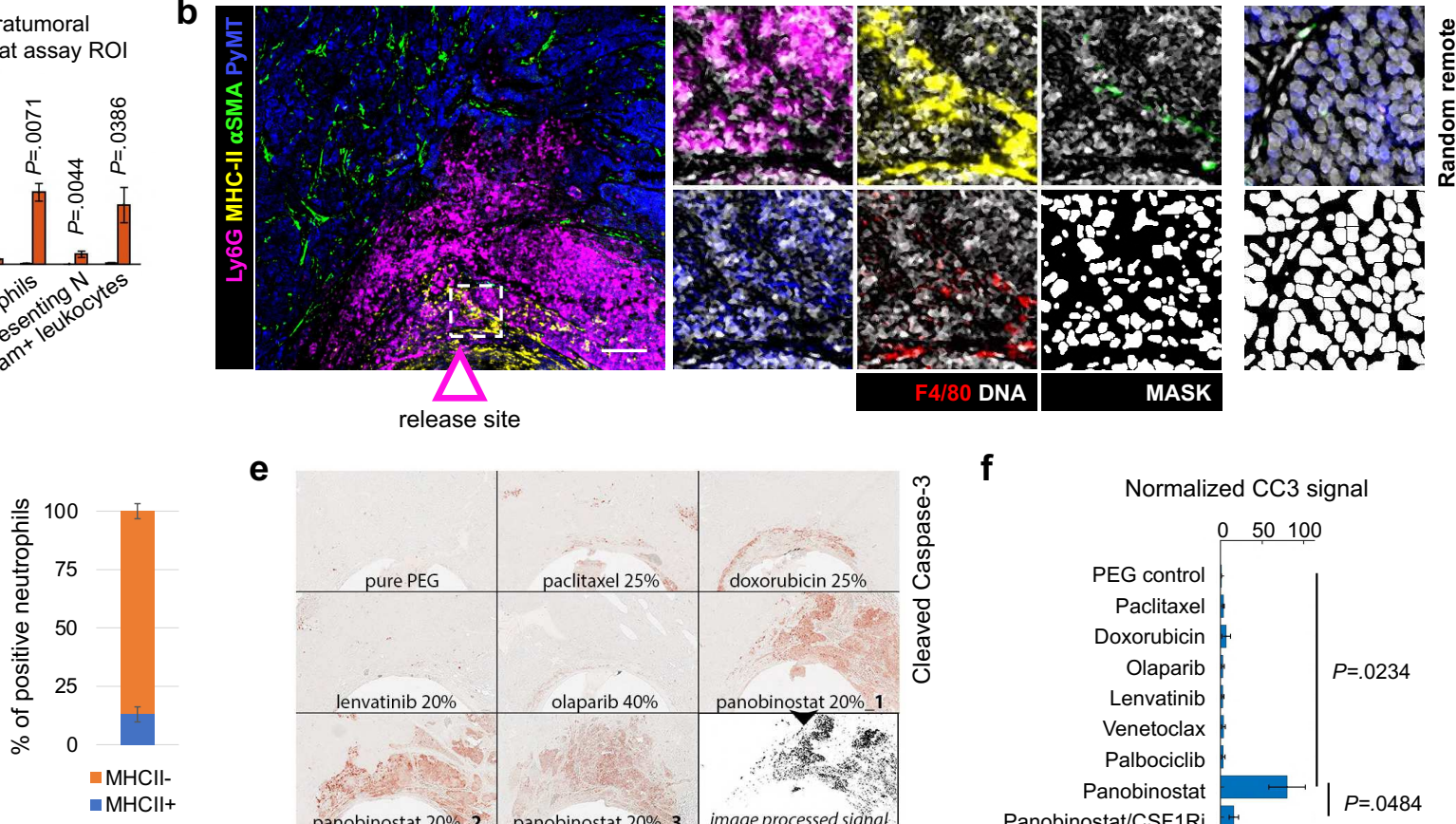

g

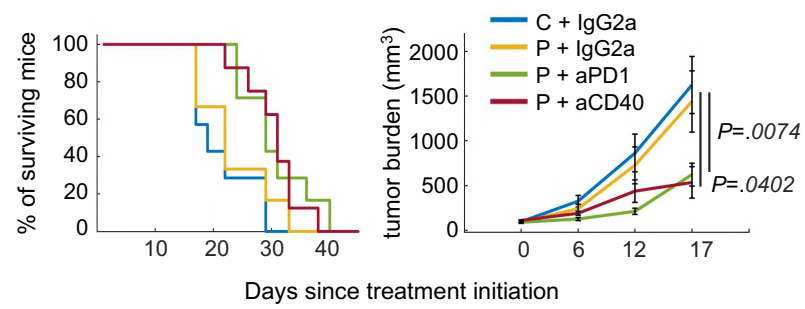

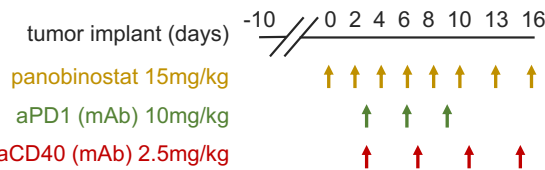

h

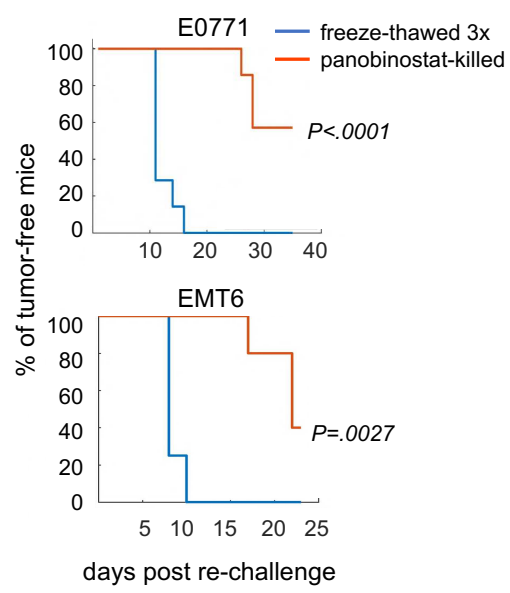

Fig. 4| Local effects of panobinostat and whole animal studies testing induction of anti-tumor immunity in mouse mammary carcinoma. a, Quantification of single cell events using individual markers and standard cell types. Bars are mean \pm s.e.; $n=3$ reservoirs. Significance was calculated by paired sample one tailed t-test. For quantification of all cell, see Extended Data Fig. 5a. b. Sample composite image of the key response markers at the panobinostat well. A dashed box defines the magnified area (right), which shows F4/80 staining in red and DNA signal and DNA-derived mask in white. Scale bar, $100 \mu \mathrm{m}$. c, Marker co-expression in XY coordinates. Each dot represents a marker positive cell. Coordinate [0,0] identifies the drug source. The direction of the drug release is upwards. $\mathbf{d}$, Percentage of MHC-II+ neutrophils. Shown is mean \pm s.e.; $n=3$ panobinostat reservoirs. e, CC 3 IHC image of a sectioned tissue surrounding the IMD at depicted targeted agents and chemotherapies. Three replicates are presented for the most potent death-inducing drug, panobinostat. A computationally processed CC3 signal is shown as a binary image. f, Quantification of PEG normalized average mean CC3 intensity ( $p x$ value) in the assay region. The graph shows mean \pm s.e; $n=3$ wells per treatment; significance was calculated using an independent two-sample t-test with equal variance. g, Survival rates (left) and tumor burden measurements (right) of mice bearing E0771 tumors after systemic treatment using control diluent (C), panobinostat (P), anti-PD1, anti-CD40 and IgG2a isotype control monoclonal antibody. Shown is mean \pm s.e.; $n=7-8$ mice per group. Significance was calculated by log-rank (Mantel-Cox) and by an unpaired two-tailed t-test with equal variance for survival and tumor burden rate, respectively. For results using anti-PD-1 and anti-CD40 monotherapy see Fig. $6 \mathrm{c}$. Treatment dose and schedule is presented. $\mathbf{h}$, Induction of anti-tumor immunity measured in a vaccination study using panobinostat treated cells and negative control (cells killed by three freeze/thaw cycles). Line graphs show percentages of mice free from palpable tumors. The P-value was calculated by log-rank (Mantel-Cox) test. $n=7$ per each group for E0771 model; and $n=4$ (control) and n=5 (experimental) for EMT6 model, respectively. 
a $\quad$ Antigen presenting macrophages

Antigen presenting neutrophils

Neutrophils

(Phagocytic) DCs

Cancer stem cells (CSC)

CD45

$\mathrm{cC3}$

Myeloperoxidase

ICAM-1

Neuropilin-1

MHC-I

Calreticulin

Galectin-3

Distance
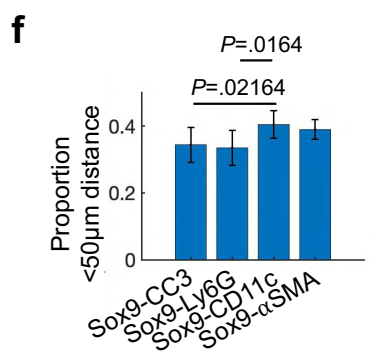

- Border

- Distal b

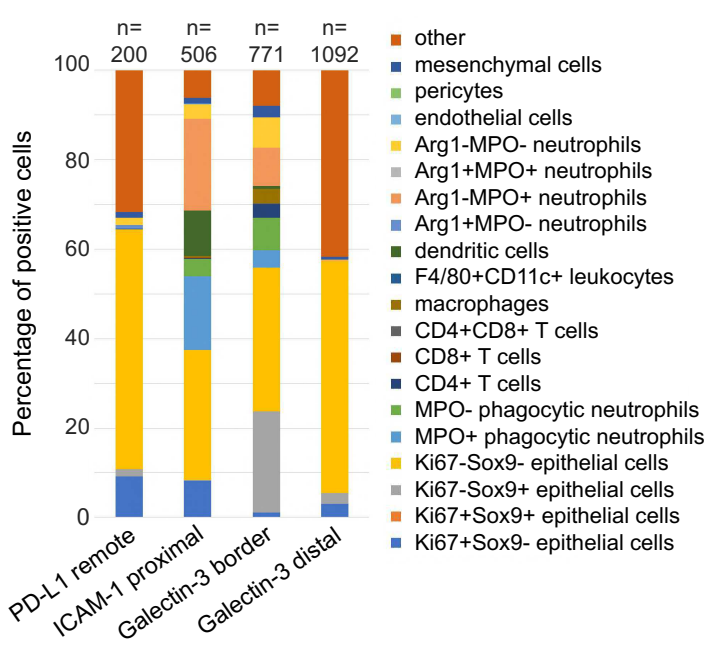

d
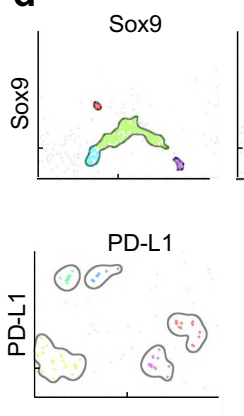

CD45
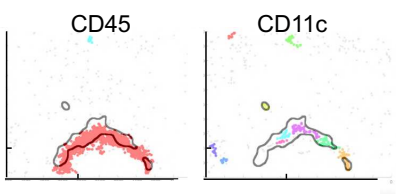

Galectin-3
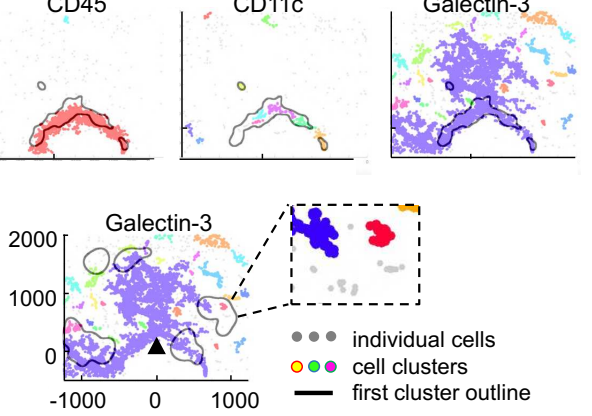

C
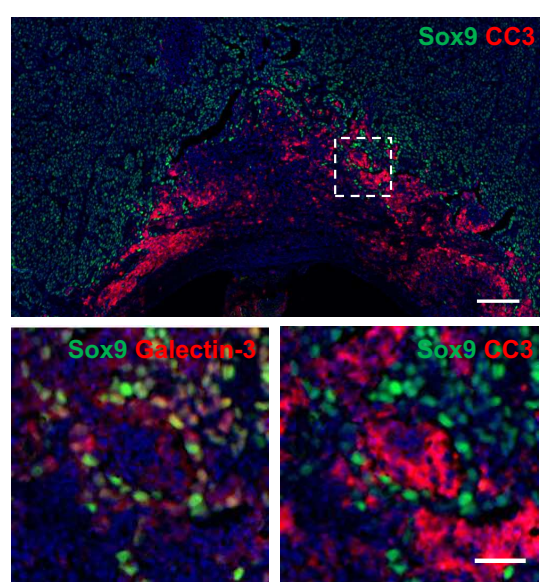

e

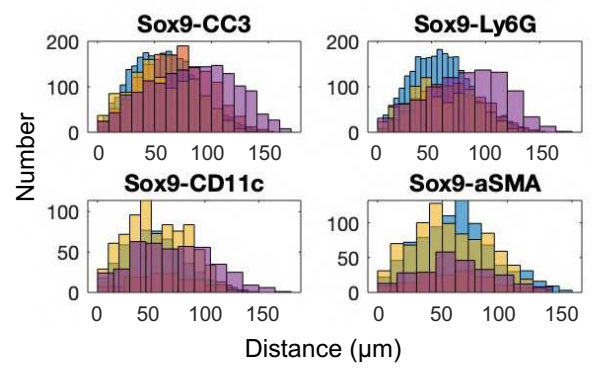

Fig. 5| Spatial single cell analyses of immunogenic cell death biomarkers and associated resistance mechanisms. a, Profile plot of the relative abundance of standard cell types and individual biomarkers with distance from the well. Assay zones are color-coded in the legend; profile area is shown in Extended Data Fig. 6a. b, Percentages of cells expressing biomarkers of ICD on standard cell types presented in form of a stack bar graph. The number of cells quantified $(n)$ is presented. c, A composite image showing mutually exclusive staining of Sox9 and CC3; and co-expression of Sox9 with galectin-3 (bottom left image). Scale bar $100 \mu \mathrm{m}$ and $25 \mu \mathrm{m}$ for top and bottom images, respectively. d, Distance-based clustering of depicted marker positive cells in XY coordinates with overlay (black line) with Sox9 (top) and PD-L1 cluster border (bottom), respectively. Individual clusters were

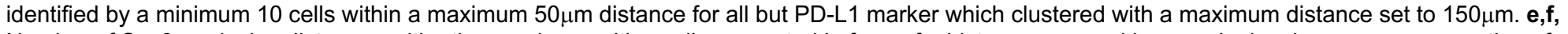
Number of Sox9+ pairwise distances with other marker positive cells presented in form of a histogram, e; and bar graph showing average proportion of Sox9 pairwise distances which were less than $50 \mu \mathrm{m}, \mathrm{h}$. $\mathrm{n}=4$ ROls of $175 \mu \mathrm{m}$ diameter in the border assay zone. Significance was determined by paired two tailed $t$ test. 
Fig. 6

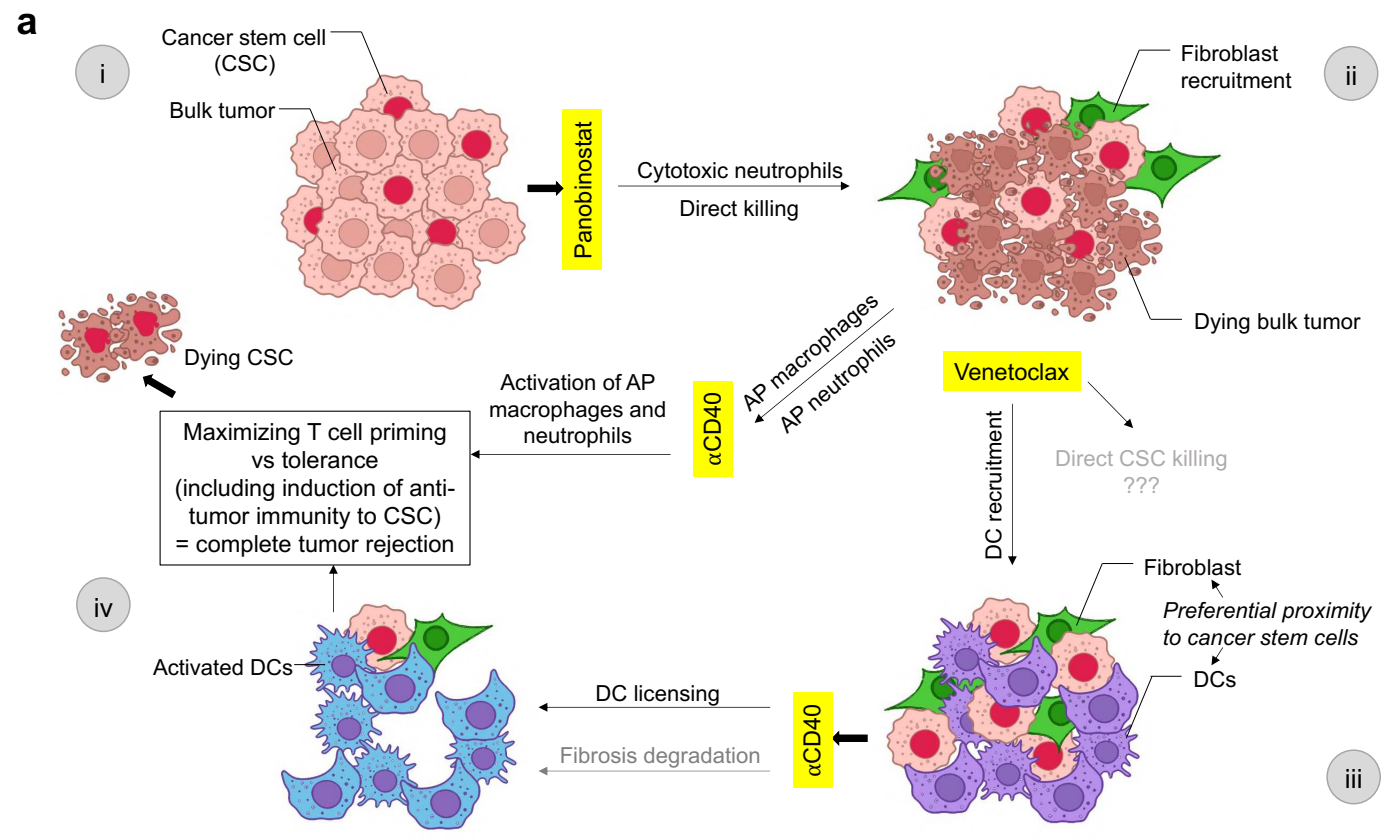

b

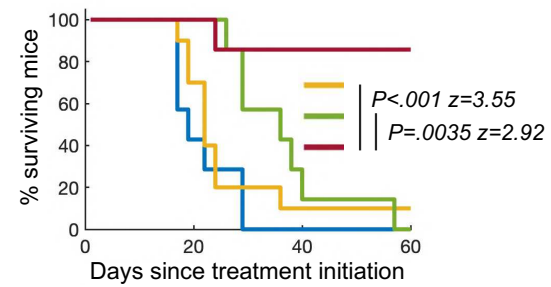

E0771

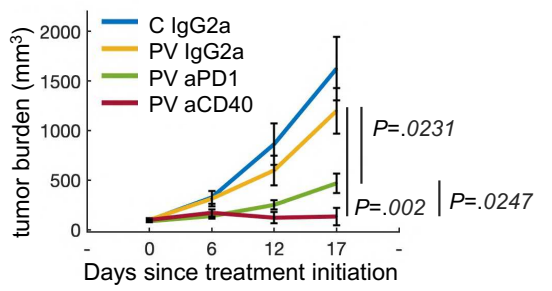

d

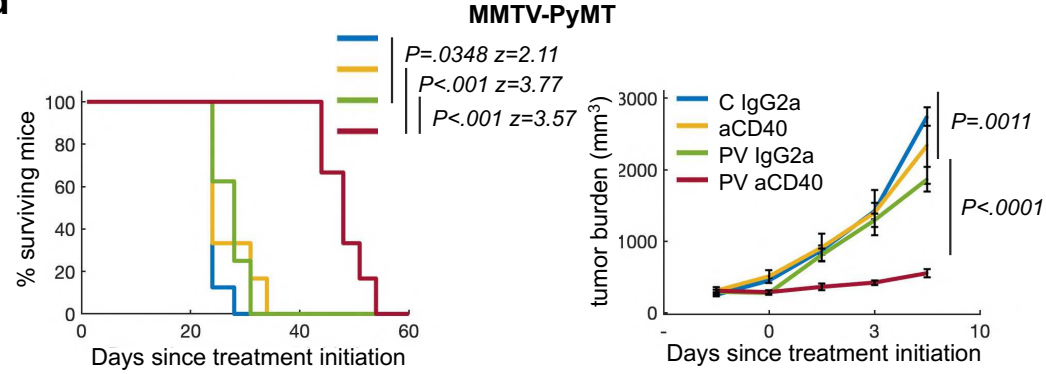

C
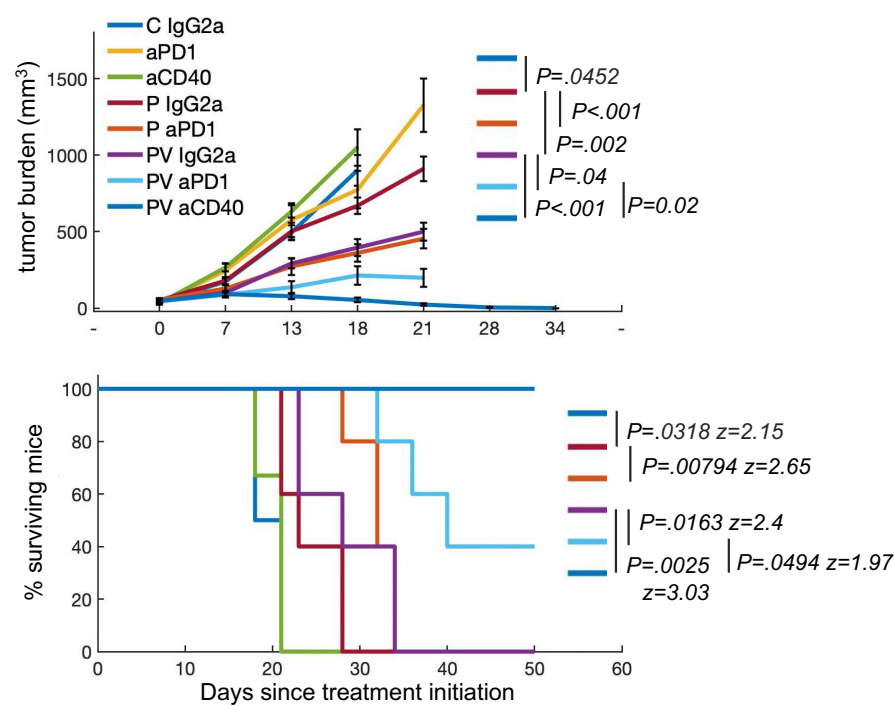

Fig. 6| Efficacy of the triple combination of panobinostat, venetoclax and anti-CD40 immunotherapy in mammary carcinoma and rationale for the combination. a, Hypothetical model of response for panobinostat/venetoclax/anti-CD40 triple combination treatment efficacy in breast cancers. Briefly, the tumor is composed of bulk tumor and cancer stem cells (i). Panobinostat induces immunogenic cell death of the bulk tumor while CSCs remain resistant in the tumor microenvironment (ii). Venetoclax induces recruitment of dendritic cells in close proximity to cancer stem cells (iii). We hypothesize that if CD40 ligation induces licensing of DCs which captured and processed antigen from neighboring CSCs, the triple combination potentiates CSC-specific anti-tumor immunity leading to complete tumor rejection (iv). b, c, d, Survival rate (left and bottom graphs; $100 \%$ to $0 \%$ ) and tumor burden measurements (right and top graphs) over time in E0771, b; EMT6, c; orthotopically induced tumor bearing mice and MMTV-PyMT mice with spontaneously growing tumors, d. C, control; P, panobinostat, PV, panobinostat-venetoclax combination. Treatment schedules and doses match those in Fig. $3 \mathrm{~g}$ and $4 \mathrm{~g}$ except the doses for panobinostat and venetoclax were decreased to $11.5 \mathrm{mg} / \mathrm{kg}$ and $18 \mathrm{mg} / \mathrm{kg}$, respectively, when drugs were combined. For survival rate, P-value was calculated by log-rank (Mantel-Cox). For tumor burden, line graphs are mean \pm s.e. per timepoint; $n=8-12$ mice, and 6-12 tumors and 6-8 mice per group in b, $c$ and d, respectively. Significance was calculated by unpaired two-tailed t-test with equal variance. 


\section{Supplementary Files}

This is a list of supplementary files associated with this preprint. Click to download.

- MIMANBTSupplementaryinformationfiles.pdf 